\title{
Shock discontinuities around the confinement-deconfinement transition in baryon-rich dense matter
}

\author{
D. H. Rischke \\ Institut für Theoretische Physik der J.W. Goethe Universität, Postfach 111932, D-6000 Frankfurt am Main 11, West Germany \\ B. L. Friman \\ Gesellschaft für Schwerionenforschung (GSI), Postfach 110552, D-6100 Darmstadt, West Germany \\ B. M. Waldhauser, H. Stöcker, and W. Greiner \\ Institut für Theoretische Physik der J. W. Goethe Universität, Postfach 111932, D-6000 Frankfurt am Main 11, West Germany
}

(Received 15 May 1989)

\begin{abstract}
We investigate shock discontinuities that involve a conversion of hadronic matter into quarkgluon matter and vice versa. Such discontinuities may develop when nuclear matter is compressed to energy densities beyond the deconfinement transition and in the hadronization of an expanding quark-gluon plasma. In these investigations we study the influence of various phenomenological equations of state. Consequences for entropy production in heavy-ion collisions are discussed and estimates of inclusive particle ratios at freezeout are given. We find that antiparticle-to-particle ratios may be enhanced by an order of magnitude if a quark-gluon plasma is created during the collision compared to a purely hadronic collision scenario.
\end{abstract}

\section{INTRODUCTION}

In nucleus-nucleus collisions at ultrarelativistic energies the properties of matter at very high energy densities can be studied. One of the primary motivations for these studies is the possibility that matter in a color deconfined phase, i.e., a quark-gluon plasma (QGP), could be formed in such collisions, ${ }^{1}$ as predicted by lattice simulations of quantum chromodynamics ${ }^{2}$ (QCD). On the theoretical side, models of the reaction dynamics have been developed, which provide a framework for studying signals for the creation of a transient plasma. Many of these models are based on the assumption that hydrodynamics applies for the dynamical evolution of a heavy-ion collision. The hydrodynamical approach is justified if the mean free path of the constituent particles is much smaller than the size of the system under consideration, which is a reasonable assumption in the case of large and dense systems.

Once one is convinced of the validity of the hydrodynamical description, the actual calculation of the reaction dynamics is simple in principle: One has to supplement the equations of relativistic hydrodynamics with a reasonable equation of state for the matter under consideration and solve them (if one is interested in dissipative phenomena one has to additionally specify transport coefficients and use the more general equations of dissipative hydrodynamics). However, numerical evaluation is rendered more difficult by the fact that a violent reaction may cause very strong gradients in all thermodynamic quantities and the matter flux velocity (in ideal hydrodynamics, these gradients may in fact be infinite). The possibility, that such discontinuities (shock waves) may occur in high-energy nuclear collisions, was discussed al- ready some time ago. ${ }^{3-6}$ Experimental data indicate $e^{4,7}$ that shock waves can indeed play a role in the reaction dynamics of a heavy-ion collision.

The mathematical treatment of these discontinuities is particularly simple in a one-dimensional steady-flow version of the equations of ideal hydrodynamics. In view of this and because of possible experimental relevance many authors have investigated physical consequences of shock discontinuities in the compression stage of a reaction $^{3-5,8-12}$ and in the hadronization phase transition between a quark-gluon plasma and hadronic matter., 12-15 However, in most publications only the case of baryonpoor matter $\left(\mu_{B}=0\right)$ is investigated or other simplifying assumptions are made.

In this paper we extend these investigations to finite baryon density and include strange degrees of freedom. Furthermore quantum statistical properties of the constituent particles are taken into account. We study general aspects of the resulting shock solutions (e.g., stability). As an application, we discuss entropy production in the shock discontinuities and the consequence for observables (particle ratios). Entropy can be produced in the compression stage of the reaction and, provided that a QGP was created, also in the expansion stage, if combustion shock fronts are formed (we neglect dissipative phenomena in the late expansion stage, immediately preceding freezeout, since a correct treatment would require more accurate knowledge of the transport properties of nuclear matter, of the actual expansion geometry, and of chemical reactions between hadrons). We show that the additional entropy produced may enhance, in turn, the antiparticle-to-particle ratio by a factor of 10 compared to a purely hadronic collision at the same bombarding energy. 
We employ various nuclear-matter equations of state. Two different phenomenological functional forms are applied for the compressional part of the hadronic energy per baryon. Both equations of state are studied in a strange as well as a nonstrange scenario, i.e., with and without strange particles in the quark and hadron phases (cf. Table I). Moreover, in some of our calculations a nonlinear $\sigma-\omega$ model $^{16}$ is used as the hadronic equation of state.

The course of the paper is the following. In Sec. II the phenomenological equations of state are presented and a simple reaction model is sketched. Section III deals with general aspects of shock phenomena in the compression stage of a collision and of combustions in the nonequilibrium phase transition between the QGP and hadronic matter. In Sec. IV the results of Sec. III are applied to calculate the entropy produced in our simple collision picture and the consequences for experimentally observable quantities at freezeout are discussed. Section $\mathrm{V}$ concludes this work with a summary of our results.

TABLE I. Hadrons and hadronic resonances used in the calculation of thermodynamic quantities of the hadronic phase with equations of state (10) and (11). The corresponding antiparticles are not shown.

\begin{tabular}{|c|c|c|c|c|c|}
\hline $\begin{array}{c}\text { Cluster } \\
i \\
\end{array}$ & $\begin{array}{c}\begin{array}{c}\text { Mass } \\
(\mathrm{MeV})\end{array} \\
\end{array}$ & $\begin{array}{c}\text { Quark } \\
\text { content }\end{array}$ & $\begin{array}{c}\text { Strange- } \\
\text { ness }\end{array}$ & $\begin{array}{c}\text { Degen- } \\
\text { eracy }\end{array}$ & $\theta_{i}$ \\
\hline 1 & 138 & 0 & 0 & 3 & -1 \\
\hline 2 & 549 & 0 & 0 & 1 & -1 \\
\hline 3 & 770 & 0 & 0 & 9 & -1 \\
\hline 4 & 783 & 0 & 0 & 3 & -1 \\
\hline 5 & 939 & 3 & 0 & 4 & 1 \\
\hline 6 & 958 & 0 & 0 & 1 & -1 \\
\hline 7 & 1232 & 3 & 0 & 16 & 1 \\
\hline 8 & 1440 & 3 & 0 & 4 & 1 \\
\hline 9 & 1520 & 3 & 0 & 8 & 1 \\
\hline 10 & 1535 & 3 & 0 & 4 & 1 \\
\hline 11 & 1620 & 3 & 0 & 8 & 1 \\
\hline 12 & 1650 & 3 & 0 & 4 & 1 \\
\hline 13 & 1675 & 3 & 0 & 12 & 1 \\
\hline 14 & 1680 & 3 & 0 & 12 & 1 \\
\hline 15 & 1700 & 3 & 0 & 24 & 1 \\
\hline 16 & 1710 & 3 & 0 & 4 & 1 \\
\hline 17 & 1720 & 3 & 0 & 8 & 1 \\
\hline 18 & 1905 & 3 & 0 & 24 & 1 \\
\hline 19 & 1910 & 3 & 0 & 8 & 1 \\
\hline 20 & 1930 & 3 & 0 & 24 & 1 \\
\hline 21 & 1950 & 3 & 0 & 32 & 1 \\
\hline 22 & 495 & 1 & -1 & 2 & -1 \\
\hline 23 & 1115 & 2 & 1 & 2 & 1 \\
\hline 24 & 1190 & 2 & 1 & 6 & 1 \\
\hline 25 & 1317 & 1 & 2 & 4 & 1 \\
\hline 26 & 1672 & 0 & 3 & 4 & 1 \\
\hline 27 & 895 & 1 & -1 & 6 & -1 \\
\hline 28 & 1405 & 2 & 1 & 2 & 1 \\
\hline 29 & 1385 & 2 & 1 & 12 & 1 \\
\hline 30 & 1530 & 1 & 2 & 8 & 1 \\
\hline 31 & 1020 & 0 & 0 & 3 & -1 \\
\hline
\end{tabular}

\section{THE NUCLEAR-MATTER EQUATION OF STATE AND A SCHEMATIC REACTION PICTURE}

A general nuclear-matter equation of state, which covers the perturbative as well as the nonperturbative regime of QCD, is still lacking. Therefore we apply a phenomenological form for the hadronic "part" of the nuclearmatter equation of state and perturbative QCD (Ref. 17) with an MIT bag pressure term ${ }^{18}$ for the QGP part. Both equations of state are amalgamated via Gibbs equilibrium conditions. In the case that no strange quarks are present the plasma part is, as usual ${ }^{17}(\hbar=c=1)$,

$$
\begin{aligned}
p q\left(T, \mu_{q}\right)= & \frac{8 \pi^{2}}{45} T^{4}\left(1-\frac{15 \alpha_{s}}{4 \pi}\right)+\frac{7 \pi^{2}}{30} T^{4}\left(1-\frac{50 \alpha_{s}}{21 \pi}\right) \\
& +\left[\mu_{q}^{2} T^{2}+\frac{\mu_{q}^{4}}{2 \pi^{2}}\right)\left(1-\frac{2 \alpha_{s}}{\pi}\right)-B,
\end{aligned}
$$

where the strong coupling constant $\alpha_{s}$ is ${ }^{19}$

$$
\alpha_{s}=\frac{4 \pi}{11-\frac{4}{3}}\left[\ln \left[\frac{0.8 \mu_{q}^{2}+15.622 T^{2}}{\Lambda^{2}}\right)\right]^{-1} \text {. }
$$

All thermodynamical quantities are obtained from the pressure $p q$ using standard relations:

$$
\begin{aligned}
& \bar{\rho}_{q}=\left(\frac{\partial p}{\partial \mu_{q}}\right)_{T}, \quad s=\left(\frac{\partial p}{\partial T}\right)_{\mu_{q}}, \\
& \bar{e}+p=T s+\mu_{q} \bar{\rho}_{q} .
\end{aligned}
$$

If we additionally have strange quarks, a term

$$
\begin{aligned}
p_{Q}^{s}\left(T, \mu_{s}\right)=\frac{3 T}{\pi^{2}} \int_{0}^{\infty} d k k^{2}[ & \ln \left(1+e^{-\beta\left(\sqrt{k^{2}+m_{s}^{2}}-\mu_{s}\right)}\right) \\
& \left.+\ln \left(1+e^{-\beta\left(\sqrt{k^{2}+m_{s}^{2}}+\mu_{s}\right)}\right)\right]
\end{aligned}
$$

is to be added to (1), and, lacking an expression for $\alpha_{s}$ for massive $\left(m_{s} \simeq 150 \mathrm{MeV}\right)$, interacting quarks at finite $T$ and $\rho$, we put $\alpha_{s}=0$ in this case. Equations (3) are modified to

$$
\begin{aligned}
& \bar{\rho}_{q}=\left(\frac{\partial p}{\partial \mu_{q}}\right)_{T, \mu_{s}}, \bar{\rho}_{s}=\left[\frac{\partial p}{\partial \mu_{s}}\right]_{T, \mu_{q}}, \\
& s=\left(\frac{\partial p}{\partial T}\right]_{\mu_{q}, \mu_{s}}, \bar{e}+p=T s+\mu_{q} \bar{\rho}_{q}+\mu_{s} \bar{\rho}_{s} .
\end{aligned}
$$

Although hadronic matter is, in contrast with the plasma, directly accessible to experiments, our knowledge of its properties is extremely vague except around the saturation state. A simple model of nuclear matter far away from the ground state is that of a quantum gas consisting of noninteracting point particles. This picture is, however, not consisten $\mathrm{t}^{20}$ with the concept of a phase transition to deconfined quarks and gluons at high temperatures ${ }^{2}$ and densities. The important feature to be accounted for is the short-range hadron-hadron interaction, which many authors ${ }^{21,22}$ introduce in the form of an eigenvolume correction following Hagedorn: ${ }^{23}$ 


$$
\text { density }=\frac{\text { density of point particles }}{1+\bar{e}^{p p} / 4 B},
$$

where $\bar{e}^{p p}$ is the energy density of hadronic pointlike particles and $4 B$ the ground-state energy density of hadrons in MIT bag model. ${ }^{18}$ The correction factor ensures that thermodynamic densities converge towards finite values while the corresponding point-particle densities approach infinity. This restrains the hadronic pressure so that the quark phase is thermodynamically stable at high $T$ and $\rho$.

However, equation of state (6) causes a problem when one tries to estimate the energy density of nuclear matter in the hot, compressed region in a relativistic heavy-ion collision. This estimate requires (a) a reasonable assumption on how much of the available bombarding energy is converted into internal (c.m.) energy per baryon $\bar{e} / \bar{\rho}_{B}$ and (b) a suitable model for the dynamics of the compression stage.

Such a model [e.g., a one-dimensional relativistic hydrodynamical shock model, i.e., the Rankine-HugoniotTaub-adiabat (RHTA) equation] relates thermodynamic variables in the compressed zone (e.g., the energy density $\bar{e}$ and the net baryon density $\bar{\rho}_{B}$ ), such that the state of the fireball becomes a unique function of, e.g., the energy per baryon $\bar{e} / \bar{\rho}_{B}$ and thus, through (a), of the bombarding energy. However both energy density and baryon density are limited for the equation of state (6), for instance, $\bar{e} \leq 4 B$. Consequently, the range of bombarding energies compatible with the equation of state (6) and the shock model is constrained. Thus, a reasonable comparison (to arbitrary bombarding energies) of some observable obtained in a "purely" hadronic shock and a shock leading to plasma formation is not possible. Another

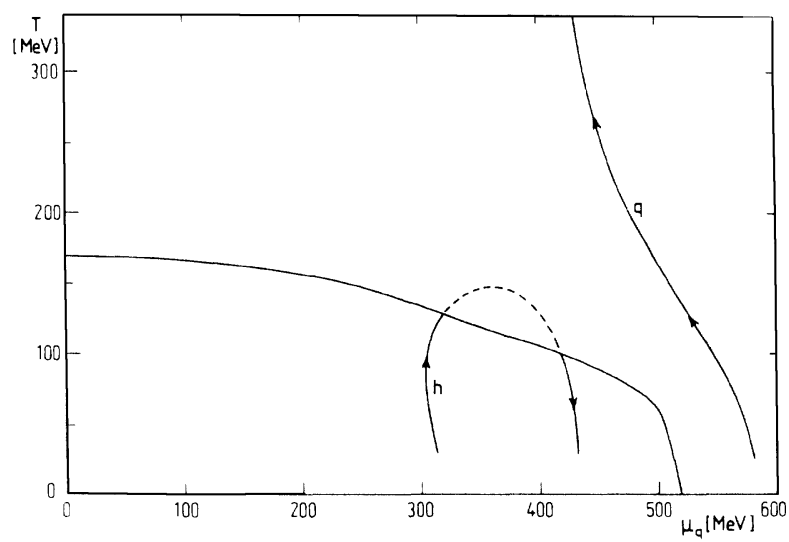

FIG. 1. Phase-transition line and RHTA's for a shock into QGP and hadron matter described by equation of state (6), $B=250 \mathrm{MeV} \mathrm{fm}^{-3}, \Lambda=100 \mathrm{MeV}$. Arrows indicate increasing bombarding energy.

consequence is the peculiar behavior of the shock adiabat shown in Fig. 1.

We therefore decided to take hadronic short-range interactions into account by means of an effective phenomenological interaction potential $U\left(\rho_{B}\right)$ acting between baryons, where $\rho_{B}$ is the net baryon density. ${ }^{9}$ This enables us to treat hadronic matter as a mixture of quantum gases consisting of point particles. To ensure consistency with general thermodynamical relations the grand potential density $\omega_{H}\left(T, \mu_{B}\right)$ (in the nonstrange case) must be of the form

$$
\omega_{H}=-p_{H}=-T \sum_{i} g_{i} \theta_{i} \sum_{\mathbf{k}} \ln \left(1+\theta_{i} \exp \left\{-\beta\left[\epsilon_{i}(\mathbf{k})-b_{i}\left(\mu_{B}-\Sigma\right)\right]\right\}\right)+\bar{u}-\Sigma \bar{\rho}_{B},
$$

where $\theta_{i}=+1(-1)$ for fermions (bosons), $g_{i}$ being the spin-isospin degeneracy of particle species $i, \epsilon_{i}(\mathbf{k})$ the corresponding relativistic, free one-particle energy $\left(\mathbf{k}^{2}+m_{i}^{2}\right)^{1 / 2}, b_{i}$ the baryon number of particle species $i$, and $\Sigma=d \bar{u} / d \bar{\rho}_{B}$, $\bar{u}=U\left(\bar{\rho}_{B}\right) \bar{\rho}_{B} / \bar{N}_{B}, \mu_{B}=3 \mu_{q}$ in chemical equilibrium for nonstrange baryons. Equation (7) is formally equivalent to the corresponding expression in the mean-field approximation of a relativistic quantum field theory. ${ }^{10}$ In the case that strange particles are present, too, strangeness is also conserved, since weak decays play no role on the time scale of a heavy-ion collision. Thus one obtains ${ }^{9}$

$$
\omega_{H}=-p_{H}=-T \sum_{i} g_{i} \theta_{i} \sum_{\mathbf{k}} \ln \left(1+\theta_{i} \exp \left\{-\beta\left[\epsilon_{i}(\mathbf{k})-z_{q}^{i}\left(\mu_{q}-\Sigma / 3\right)-z_{s}^{i}\left(\mu_{s}-\Sigma / 3\right)\right]\right\}\right)+\bar{u}-\Sigma \bar{\rho}_{B},
$$

where $z_{s}^{i}$ and $z_{q}^{i}$ are the strange and nonstrange (net) quark content of hadronic species $i$. Note that, although the baryonic interaction $\Sigma$ seems to modify both chemical potentials $\mu_{q}, \mu_{s}$, there is no effect on strange mesons. To make this clear, rewrite

$z_{q}^{i}\left(\mu_{q}-\Sigma / 3\right)+z_{s}^{i}\left(\mu_{s}-\Sigma / 3\right)=z_{q}^{i} \mu_{q}+z_{s}^{i} \mu_{s}-\frac{1}{3}\left(z_{q}^{i}+z_{s}^{i}\right) \Sigma$,

and note that $\frac{1}{3}\left(z_{q}^{i}+z_{s}^{i}\right) \equiv b_{i}$ which vanishes for all mesons. Thus in this model, e.g., kaon condensation sets in when $\mu_{q}-\mu_{s} \geq m_{K}$. The consequences, e.g., for the phase boundary between quark and hadron matter are discussed in Ref. 24.

The potential $U\left(\bar{\rho}_{B}\right)$ is chosen so that the properties of nuclear matter are reproduced. To retain a close connection to the concept of compressional energy in nuclear physics $^{25}$ we parametrize $U$ in the following way: ${ }^{9}$

$$
\frac{U}{\bar{N}_{B}}\left(\bar{\rho}_{B}\right)=W_{C}\left(\bar{\rho}_{B}\right)-W_{\mathrm{kin}}\left(\bar{\rho}_{B}, T=0\right)+W_{R},
$$

where $W_{C}$ is the compressional part of the total energy per baryon, $W_{R}$ the rest-mass part, and $W_{\text {kin }}\left(\bar{\rho}_{B}, T=0\right)$ 
the kinetic energy and rest mass per baryon at $T=0$ (the latter two are approximated by using only the nucleonic expressions $^{26}$ ). For alternative parametrizations, see, e.g., Refs. 10, 24, and 27. Equation (9) is constructed in a way that for $T=0, \bar{E} / \bar{N}_{B} \equiv W_{C}+W_{R}$. In order to fix nuclear-matter properties, one has to make an appropriate choice of $W_{C}$. We consider two different phenomenological functional forms for $W_{C}$. The first is the wellknown ansat $z^{28}$

$$
W_{C}=\left\{\begin{array}{l}
\frac{C}{18 \rho_{0} \bar{\rho}_{B}}\left(\bar{\rho}_{B}-\rho_{0}\right)^{2}+B_{0}, \quad \bar{\rho}_{B}>\rho^{\prime}, \\
a \bar{\rho}_{B}, \quad \bar{\rho}_{B} \leq \rho^{\prime},
\end{array}\right.
$$

$\rho^{\prime}=\rho_{0} /\left(1-9 B_{0} / C\right), a=C\left[1-\left(\rho_{0} / \rho^{\prime}\right)^{2}\right] / 18 \rho_{0}, \rho_{0}=0.17$ $\mathrm{fm}^{-3}, B_{0}=-16 \mathrm{MeV}$. This equation of state has the advantage that it is causal at all densities, i.e., the velocity of sound is less than the velocity of light, ${ }^{29}$ $c_{s}=\sqrt{(\partial p / \partial \bar{e})_{\sigma}}<1$. However, in order to get similar stiffness in the high-density region as in meson fieldtheoretical models ${ }^{16}$ one is forced to choose values for $C$ in the range $800 \mathrm{MeV} \leq C \leq 5000 \mathrm{MeV} .{ }^{9}$ A value of $C$ in this range yields a compressional energy in rough agreement with intermediate-energy heavy-ion experiments. ${ }^{30}$ Nevertheless, at low densities the equation of state is much too stiff; the empirical value for the incompressibility at the saturation point is $K_{0}=200-300 \mathrm{MeV}$ (Refs. 31 and 32), while for the above equation of state $K_{0} \equiv C \gg 300 \mathrm{MeV}$. Thus, such a simple ansatz cannot describe nuclear matter both at high and low densities. However, as was argued in Ref. 9, if one restricts oneself to the hadron-matter-QGP phase transition, i.e., to high densities and/or high temperatures, the equation of state (10) is reasonable, ${ }^{33}$ with an appropriate choice of the constant $C$.

Nevertheless, another flaw of (10) will become evident when calculating the sound speed $c_{s}$ (cf. Appendix) in order to determine the Chapman-Jouguet (CJ) point (see Sec. III). This involves the second derivative of $W_{C}$, which is not continuous at $\bar{\rho}_{B}=\rho^{\prime}$. Hence $c_{s}$ is not continuous, which leads to ambiguities (cf. Figs. 5 and 6).

Consequently, Eq. (10) is expected to yield a good description of hadronic matter at high densities, while the low-density behavior is not well reproduced. We also study the consequences of a new ansatz,

$$
W_{C}(x)=16 \mathrm{MeV}\left[\mathcal{K}\left[x+\frac{(1+\mathcal{K})^{2}}{x+\mathcal{K}}\right]-(1+\mathcal{K})^{2}\right] \text {, }
$$

which is well behaved at low densities. In Eq. (11) $x=\bar{\rho}_{B} / \rho_{0}$, where $\rho_{0}$ is the ground-state density and $\mathcal{K}$ is a dimensionless parameter, which controls the stiffness of the equation of state. The ground-state incompressibility of this equation of state is

$$
K_{0}=288 \mathrm{MeV} \frac{\mathcal{K}}{1+\mathcal{K}} .
$$

Equation (12) implies that $K_{0}$ cannot exceed $288 \mathrm{MeV}$ for positive $\mathcal{K}$. For negative $\mathcal{K}$ again the absolute minimum of the energy of nuclear matter is not at $\rho_{0}=0.17 \mathrm{fm}^{-3}$.
The equation of state (11) has the advantage that it is continuously differentiable for all $x$ and, depending on the value of $\mathcal{K}$, arbitrarily "stiff' at high $\bar{\rho}_{B}$, while it simultaneously allows for reasonable values of $K_{0}$. Thus, in this respect it can compete with the common linear and nonlinear meson field theories. ${ }^{16}$ The disadvantage of (11) is that for high $x$ (and $T=0$ ) the sound speed squared

$$
\begin{aligned}
c_{s}^{2} & =\left[\frac{\partial p}{\partial \bar{e}}\right]_{\sigma}(T=0)=\frac{d p / d x}{d \bar{e} / d x} \\
& =\frac{32 x \mathcal{K}\left[1-\mathcal{K}(1+\mathcal{K})^{2} /(y x+\mathcal{K})^{3}\right]}{16\left\{\mathcal{K}\left[2 x+\mathcal{K}\left(\frac{1+\mathcal{K}}{x+\mathcal{K}}\right)^{2}\right]-(1+\mathcal{K})^{2}\right\}+939}
\end{aligned}
$$

exceeds the causal limit, if $\mathcal{K}>\sqrt{939} / 4-1 \simeq 6.66$ or $K_{0}>288(1-4 / \sqrt{939}) \mathrm{MeV} \simeq 250.4 \mathrm{MeV}$. The value of $x$, at which this happens, decreases with increasing stiffness of the equation of state, i.e., with increasing $\mathcal{K}$. For $\mathcal{K}<\sqrt{939} / 4-1$ the ansatz $(11)$ is causal for all $x$. In Fig. 2(a) we compare the ansatz (11) with (10) and in Fig. 2 (b) we show $c_{s}^{2}$ as a function of $x$ for various values of $\mathcal{K}$. Note that the tiny region at low densities where the speed of sound is imaginary $\left(d p / d \bar{\rho}_{B}<0\right)$, corresponds to the well-known instability with respect to formation of droplets.

In order to obtain a stiffness of the equation of state at high densities comparable to that of typical meson field theories ${ }^{35}$ we restrict ourselves to $\mathcal{K}$ values between 4.875 and 12.125, which corresponds, in the nonlinear $\sigma-\omega$ meson field theory, to values of the vector coupling constant $c_{V}$ between 10 and 17 [or, in the ansatz (10), to 1400 $\left.\mathrm{MeV} \leq C \equiv K_{0} \leq 3500 \mathrm{MeV}\right]$. With this choice of parameters the ground-state incompressibility lies in the range $239 \mathrm{MeV} \leq K_{0} \leq 266 \mathrm{MeV}$ for (11). For the values of $\mathcal{K}$ used in most of the following $(\mathcal{K} \simeq 6.25)$, noncausality is never a problem. For $\mathcal{K}>6.66$ the ansatz (11) becomes noncausal, but for the corresponding value of $x$, at which this happens, we expect to be well inside the region where the QGP is thermodynamically stable. Only for $\mathcal{K} \gtrsim 12$ is this value of $x$ of the order of the phase transition density [cf. Figs. 2(b) and (c)]. Thus, in some sense Eqs. (10) and (11) complement each other, the first one being more realistic at high densities, the second one at low densities. For comparison some of our calculations were also done with the common nonlinear version of the $\sigma-\omega$ field theory ${ }^{16}$ as equation of state for hadronic matter.

Let us now outline a simple picture of a heavy-ion collision: the two nuclei collide forming a hot, dense zone of nuclear matter which might contain a blob of quarkgluon plasma, provided the bombarding energy is sufficiently high. We suppose that the formation of this zone can be approximated by a hydrodynamic process, i.e., a compression shock wave. Since we further assume that there is neither heat exchange with the surroundings nor evaporation of particles, the fireball, which is supposed to be in global thermodynamical equilibrium, expands isentropically until freezeout (we also neglect dissipative phenomena in the last stage of the hydrodynamical 

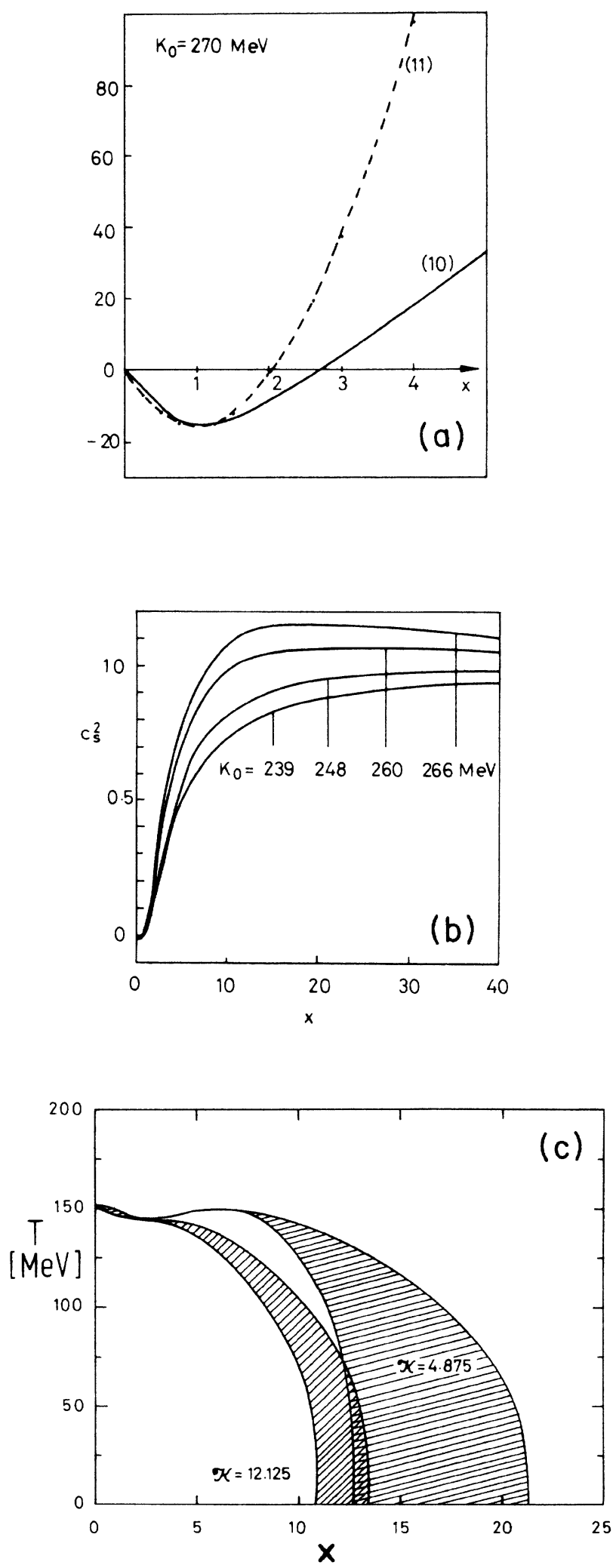

FIG. 2. (a) Equation of state (11) compared to (10) at the same ground-state incompressibility $K_{0}=270 \mathrm{MeV}$. (b) The velocity of sound of equation of state (11) as a function of $x=\bar{\rho}_{B} / \rho_{0}$ for $K_{0}=239,248,260,266 \mathrm{MeV}(\mathcal{K}=4.88,6.2,9.28$, 12.09). (c) $T-x$ diagram for the equilibrium phase transition between QGP and hadron matter with compressional energy (11).

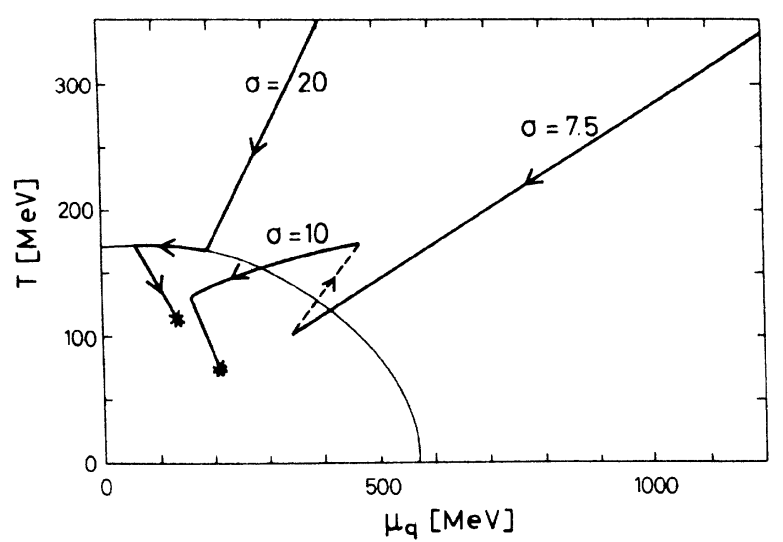

FIG. 3. Schematic $T-\mu$ diagram displaying possible expansion paths of a fireball consisting of a QGP. A nonequilibrium hadronization phase transition during the expansion, i.e., a combustion shock $\left(\sigma_{Q}=7.5 \rightarrow \sigma_{H}=10\right)$, is compared to an equilibrium phase transition $(\sigma=20=$ const $)$ along the phase coexistence curve. It is assumed that the QGP is supercooled and that the shock ends in superheated hadronic matter. Stars mark the freezeout of the system in the hadronic phase.

expansion). However, if the fireball initially contains some quark matter rather than pure hadronic matter, additional entropy can be produced in the hadronization of the plasma, which may be a nonequilibrium process, e.g., spontaneous combustion. The combustion proceeds through propagation of a shocklike discontinuity. In Fig. 3 we sketch this process in comparison with an equilibrium phase transition. ${ }^{22}$ The mathematical description of such a combustion front is identical with that of the compression discontinuity, where the plasma is created, the only difference being that the equations of state of matter in the initial and final state of the shock have to be exchanged. It should be emphasized that no shock is possible in the expansion of ordinary hadronic matter, ${ }^{36}$ i.e., when we assume that no QGP was created. We note at this point that we do not consider hadronizing shocks going into or out of the so-called mixed phase, ${ }^{12,37}$ where hadronic matter and the QGP coexist.

Let us now briefly discuss a possible consequence of the above model for heavy-ion collisions. It is well known ${ }^{36}$ that any physical shock must produce entropy because of highly dissipative processes inside the front of the shock discontinuity. This is the case in the compression stage, independently of whether a plasma is created or the system remains within the hadronic phase. However, the amount of entropy created does depend on the equation of state of the final (shocked) state. Since entropy is approximately conserved during the expansion stage, a significant difference between, e.g., the plasma and the hadronic equation of state would thus lead to a different chemical constitution of the system at freezeout. Consequently, the observed particle abundancies may give information on the initial state of the fireball, i.e., whether or not a plasma was created. Moreover, if we have a nonequilibrium phase transition between the plasma and hadrons by means of a combustion shock, additional en- 
tropy is created, probably enhancing the effect on the particle abundancies at freezeout.

\section{SHOCK DISCONTINUITIES IN THE COMPRESSION AND REHADRONIZATION STAGE}

In this section we study the properties of shock discontinuities in the compression and hadronization stage in the framework of ideal relativistic hydrodynamics. We confine our investigations of the shock front to onedimensional steady flow. In that case, thermodynamic quantities on both sides of the shock front are related by the RHTA equation ${ }^{38}$

$$
\left(X_{1} \bar{\rho}_{B, 1}\right)^{2}-\left(X_{2} \bar{\rho}_{B, 2}\right)^{2}+\left(p_{2}-p_{1}\right)\left(X_{1}+X_{2}\right)=0,
$$

which follows from conservation of energy, momentum and net baryon number across the discontinuity. Here $X=(\bar{e}+p) / \bar{\rho}_{B}^{2}$ is the generalized volume. The RHTA equation in this form is well defined only for finite net baryon density (however, an analogous equation for $\bar{\rho}_{B}=0$ was derived in Ref. 39 ). Let us first consider nonstrange quark and hadron matter. For calculating the compression shock, matter flowing into the shock front [denoted by, e.g., subscript 1 in (14)] is assumed to be in the ground state of nuclear matter, $p_{1}=0, \bar{\rho}_{B, 1}=\rho_{0}=0.17$ $\mathrm{fm}^{-3}, X_{1}=5429 \mathrm{MeV} \mathrm{fm}^{3}$, while matter in the final, "shocked" state $\left(p_{2}, \bar{\rho}_{B, 2}, X_{2}\right)$ is either excited hadronic matter or quark matter. The final shock state is determined by employing the corresponding equation of state and by fixing the bombarding energy per baryon, $E_{\text {lab }}^{\text {kin }}$, in the laboratory. The laboratory energy is related to the c.m. energy per baryon in the shocked state, $\bar{E}_{2} / \bar{N}_{B, 2}$, (assuming no energy loss through flow phenomena) via ${ }^{40}$

$$
E_{\text {lab }}^{\mathrm{kin}}=2 m_{0}\left[\left(\frac{\bar{E}_{2}}{\bar{N}_{B, 2} m_{0}}\right)^{2}-1\right],
$$

$m_{0}=923 \mathrm{MeV}$ being the rest energy per baryon in the ground state of nuclear matter. The results of the compression shock calculations are shown in Fig. 4, for hadrons $(h)$, quark matter $(q)$, and a mixture $(m)$ of both phases (see comments below) in the final shock state.

In the scenario where hadronic matter is in the final state we have a simple compression shock, without a change of the equation of state across the discontinuity. In the case of quark matter in the final state the chemical constitution is changed inside the shock front. This process is endothermic, in contrast with a combustion discontinuity, where quark matter is converted into hadronic matter (see below).

Since the equations of state of the two phases show no abnormalities, a stability analysis of the shock yields stable shocks for all relevant bombarding energies. For the hadronic shock this is immediately clear by inspection of Fig. 4 and by applying, for instance, the stability criteria given in Ref. 41. These criteria can be derived from the condition ${ }^{36}$

$$
\left[\frac{\partial^{2} p}{\partial X^{2}}\right]_{\sigma}>0
$$

along the compression Taub adiabat. The stability of the RHTA for a shock from the ground state of nuclear matter into a QGP was already investigated in Ref. 11.

Many authors have considered shocks into a mixed phase of hadron and quark matter during the compression stage of the collision. ${ }^{10,12}$ The mixed phase is thought to consist of many small bubbles of hadronic matter in the plasma or vice versa. The length scale of the inhomogeneities is assumed to be small compared to
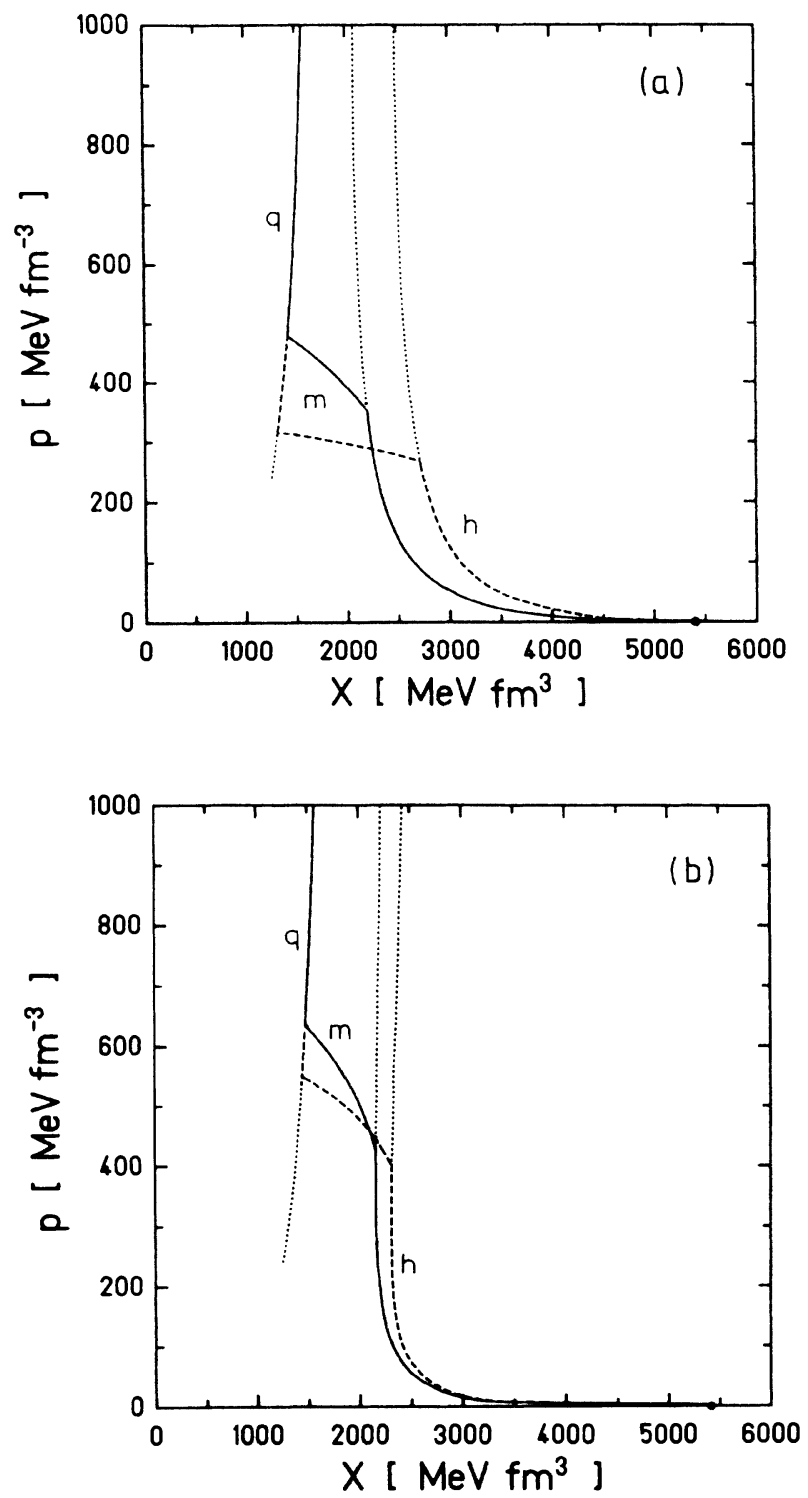

FIG. 4. RHTA's for hadronic $(h)$ and quark matter $(q)$, and a mixed phase $(m),(a)$ for equation of state $(10)$ and $B=250$ $\mathrm{MeV} \mathrm{fm}^{-3}, \Lambda=0$ : solid line, $C=1200 \mathrm{MeV}$; dashed, $C=1800$ $\mathrm{MeV}$. Dotted lines for the parts of the RHTA's, which correspond to superheated hadrons and supercooled QGP, respectively. (b) As in (a), for equation of state (11) and $B=250$ $\mathrm{MeV} \mathrm{fm}^{-3}, \Lambda=0 \mathrm{MeV}$. Full line, $\mathcal{K}=6.25$; dashed line, $\mathcal{K}=9$. Dotted lines as in (a). 
the size of the system, so that the mixed phase can be treated as a homogeneous mixture. However, for coexisting quarks and hadrons this length scale is on the order of the nuclear dimension, since the bubbles must contain several quanta to ensure thermodynamical equilibrium. Furthermore, the thickness of the shock front into a mixed phase must exceed the size of the inhomogeneities, to allow thermodynamic equilibrium to be established behind the shock. On the other hand, the thickness of the shock front must be less than the size of the system, otherwise it cannot be treated as a sharp discontinuity. ${ }^{36}$ Thus, the concept of a mixed phase, and in particular shock waves into a mixed phase, seem to be of limited value in nuclear collisions. ${ }^{42}$ Nevertheless, to make contact with related calculations, ${ }^{10,12}$ we also study this case.

The stability of such shocks against decay into double shocks ${ }^{45}$ depends crucially on the compressional part of the hadron matter equation of state. Gorenstein et al. ${ }^{10}$ found that with the equation of state proposed in Ref. 27 one obtains stable single shocks into the whole mixed phase regime, while for the linear Walecka model shock splitting occurs for shocks going into the phase transition region. In general we find that the stability of shock waves in the mixed phase is governed by the stiffness of the hadron equation of state (Fig. 4); for a sufficiently stiff equation of state the mixed phase emerges from the shock front with a velocity exceeding $c_{s}^{\text {mixed }}$, which implies that the shock is unstable. Thus, for the equation of state (10), which is hard and resembles the Walecka model, shock splitting occurs [Fig. 4(a)], while for a sufficiently soft equation of state, e.g., (11) with $\mathcal{K}=6.25$, single shocks are always stable, as for the equation of state of Ref. 27 [cf. Fig. 4(b)]. For a harder version of the equation of state (11), e.g., $\mathcal{K}=9$, a part of the mixed phase Taub adiabat becomes unstable with respect to shock splitting [Fig. 4(b)].

A mixed-phase adiabat can help to determine the bombarding energy, at which stable single shocks into the QGP become possible (via the usual geometrical construction in the $p-X$ diagram $^{45}$ or via the criterion of Ref. 41). At lower bombarding energies ( $E_{\mathrm{lab}}^{\mathrm{kin}} \sim 1-15$ $\mathrm{GeV} /$ nucleon) one should allow for the possibility of double shock formation (the particular range of energies, where shock splitting occurs, depends on the equation of state). However, we will not further pursue this question and restrict ourselves to the investigation of single shocks [we checked that for almost all sensible parameter choices one has stable single shocks into the QGP for bombarding energies in the range of interest (15-200 $\mathrm{GeV} /$ nucleon)].

A technical remark concerning the calculation of the mixed-phase adiabat should be added: An additional parameter, the volume fraction occupied by the QGP, $\delta=V_{Q} /\left(V_{Q}+V_{H}\right)$, has to be specified to compute thermodynamic densities in the mixed phase:

$$
\begin{aligned}
& \bar{e}_{2}=\bar{e}_{Q} \delta+\bar{e}_{H}(1-\delta), \\
& \bar{\rho}_{B, 2}=\bar{\rho}_{Q} \delta+\bar{\rho}_{H}(1-\delta), \ldots .
\end{aligned}
$$

To determine this additional parameter one has to solve the Gibbs phase equilibrium condition

$$
p_{Q}\left(T_{2}, \mu_{B, 2}\right)=p_{H}\left(T_{2}, \mu_{B, 2}\right)
$$

in addition to (14). Inserting (17) and (18) into (14) and setting $p_{1}=0, \bar{\rho}_{B, 1}=\rho_{0}, X_{1}=\bar{e}_{1} / \bar{\rho}_{B, 1}^{2}=e_{0} / \rho_{0}^{2}$ yields a quadratic equation for $\delta$ with the solution

$$
\begin{aligned}
& \delta=-\frac{b}{2 a}\left[1 \pm\left(1-\frac{4 a c}{b^{2}}\right]^{1 / 2}\right], \\
& a=\left(\bar{e}_{Q}-\bar{e}_{H}\right)^{2}-\left(\tilde{\rho}_{Q}-\tilde{\rho}_{H}\right)^{2}, \\
& b=p_{Q}\left(\bar{e}_{Q}-\bar{e}_{H}\right)+2\left[\bar{e}_{H}\left(\bar{e}_{Q}-\bar{e}_{H}\right)-\tilde{\rho}_{H}\left(\tilde{\rho}_{Q}-\tilde{\rho}_{H}\right],\right. \\
& c=\bar{e}_{H}^{2}-\tilde{\rho}_{H}^{2}+\bar{e}_{H} p_{Q}, \\
& \tilde{\rho}_{i}=\frac{\bar{\rho}_{B, i}}{\rho_{0}} \sqrt{e_{0}\left(e_{0}+p_{Q}\right)}, \quad i=Q, H .
\end{aligned}
$$

Having determined the equilibrium phase boundary via (18) one inserts the obtained quantities $p_{Q}, \bar{e}_{Q}, \bar{\rho}_{B, Q}, \bar{e}_{H}$, $\bar{\rho}_{B, H}$ along the phase boundary into (19). All real $\delta$ that are between 0 and 1 correspond to physical solutions of the RHTA Eq. (14) in the mixed phase.

We briefly sketch the procedure when also strange particles are considered. Then the net strangeness flux through the discontinuity $j_{s}=\bar{\rho}_{s} v \gamma$ is conserved in addition to the net baryon, energy, and momentum current. Since the ground state of nuclear matter contains no strangeness, $\bar{\rho}_{s} \equiv 0$ in any final state of the compression shock. In this case, the effect on the RHTA is small (cf. Fig. 11 below).

After presenting general aspects of compression shocks in relativistic heavy-ion collisions we now proceed with the evolution of a collision and assume that the QGP expands adiabatically along trajectories in the $T-\mu$ plane (Fig. 3) conserving the entropy produced in the compression stage (cf. Figs. 10 and 11). Combustion discontinuities may occur at the boundary of the QGP, where the plasma is converted into hadronic matter. ${ }^{13,15}$ Since the hydrodynamic expansion time sale is comparable to that of hadronization processes $\left(\delta t_{\text {had }} \sim \Lambda^{-1} \simeq 1 \mathrm{fm}\right)$ the plas$\mathrm{ma}$ is probably supercooled appreciably before hadronization sets in.

We assume that, once the hadronization of the plasma is completed by means of a combustion shock front, ${ }^{46}$ the system expands isentropically until freezeout. Note that the entropy does not increase substantially during the expansion, even if the assumption of chemical equilibrium is relaxed. ${ }^{14,47}$

Formally the mathematical description of the combustion process is completely equivalent to that of the compression stage, except that now QGP is in the "unshocked" and hadron matter in the "shocked" state. Nevertheless there are two major differences between these processes.

(a) The initial state of the combustion discontinuity on the QGP isentrope $\sigma_{Q}=$ const is determined by the dynamics of the problem. In other words, the degree of supercooling of the plasma depends on the relative size of the hadronization and expansion time scales. For the compression-shock problem in heavy-ion collisions, the system is initially in the ground state of nuclear matter. 
(b) The final state of the combustion discontinuity in the hadronic phase is in general not uniquely determined by the boundary conditions. For compression shocks, the final state is determined by the collision energy and the initial state of the matter.

Thus, within the framework of ideal hydrodynamics, we can only determine which discontinuities are consistent with conservation laws and stability criteria, but not whether they will actually develop. To study the actual evolution of such a collision, and in particular the possible development of discontinuities, a dynamical calculation, including relaxation effects, is needed.

If a certain initial shock state on the plasma isentrope $\sigma_{Q}$ is given, the physically allowed, mechanically stable spacelike $^{48}$ solutions of the corresponding RHTA equation for hadronic matter must satisfy the conditions ${ }^{36}$

$$
\begin{aligned}
& \text { (I) } \infty>j^{2}=\left(\bar{\rho}_{B} v \gamma\right)^{2} \geq 0, \\
& \text { (II) } \Delta \sigma=\sigma_{H}-\sigma_{Q} \geq 0, \\
& \text { (IIIa) } v_{Q} \geq c_{s, Q}, \quad v_{H} \leq c_{s, H} \quad \text { (detonation shocks), }
\end{aligned}
$$

or

(IIIb) $v_{Q} \leq c_{s, Q}, \quad v_{H} \leq c_{s, H} \quad$ (deflagration shocks),

where

$$
v_{Q, H}^{2}=\frac{\left(p_{Q}-p_{H}\right)\left(p_{Q, H}+\bar{e}_{H, Q}\right)}{\left(\bar{e}_{Q}-\bar{e}_{H}\right)\left(p_{H, Q}+\bar{e}_{Q, H}\right)} .
$$

For each point on the QGP isentrope $\sigma_{Q}$ one consequently finds a region of physically accessible shock states in the hadronic phase. As noted above, there is in general no definite final state for a given set of boundary conditions. However, often a unique steady-state solution, corresponding to the Chapman-Jouguet point, can be found, ${ }^{15,45}$ but it is not clear whether, in a heavy-ion collision, there is sufficient time available for such a solution to develop.

Let us note that, in the presence of additional strange degrees of freedom, the value of the net strangeness per baryon, $(S / A)_{Q}=\left(\bar{\rho}_{s} / \bar{\rho}_{B}\right)_{Q}$, in the initial state of the shock must also be specified. Furthermore, in addition to solving (14) one has to ensure the conservation of the net strangeness flux $j_{s}$ across the discontinuity. In our calculations we can simply put $(S / A)_{Q}=j_{s}=0$, since we do not consider effects of, e.g., kaon evaporation from the surface of the fireball. ${ }^{50}$

In the following we will first investigate possible hadronization discontinuities in general. In Sec. IV we will then present an illustrative calculation to estimate the entropy production in a combustion shock discontinuity.

According to (20) we find, for a given RHTA centered at $\left\{\sigma_{Q}, T_{Q},(S / A)_{Q}\right\}$, the points, where $j^{2}=\infty, j^{2}=0$, $\Delta \sigma=0$, and $v_{H}=c_{s, H}$, i.e., the points which form the boundaries of the physically allowed regions for spacelike hadronization shocks. The procedure is then repeated for every QGP state on the isentrope $\sigma_{Q}$. The physically allowed regions in the $\bar{e}_{Q}-\bar{e}_{H}$ plane ${ }^{13}$ are shown in Fig. 5. Here a RHTA is a line parallel to the $\bar{e}_{H}$ axis. We note that $j^{2}=\infty$ implies, according to the definition of $j^{2}$

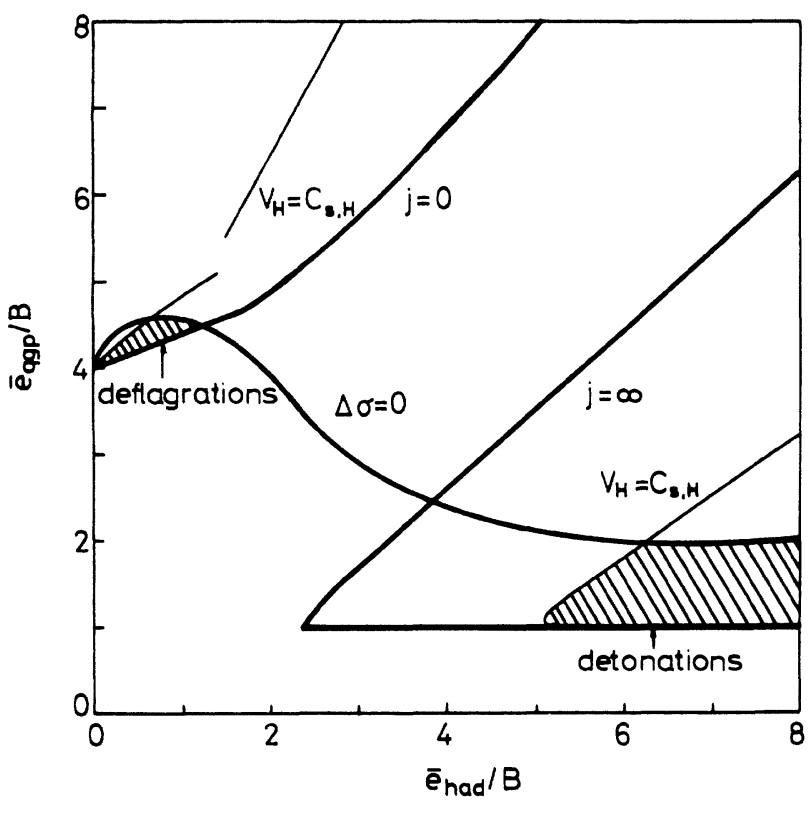

FIG. 5. $\bar{e}_{Q}-\bar{e}_{H}$ diagram illustrating the physically allowed regions of spacelike detonations and deflagrations [equation of state (10) with $C=2650 \mathrm{MeV}, B=208 \mathrm{MeV} \mathrm{fm}^{-3}, \Lambda=0$, $\sigma_{Q}=15$, without strange particles]. Note the discontinuity in the $\mathrm{CJ}$ line $v_{H}=c_{s, H}$ near the deflagration region. As discussed in Sec. II, this is caused by the fact that the ansatz (10) is not twice continuously differentiable at $\bar{\rho}_{B}=\rho^{\prime}$.

(Ref. 36), $X_{Q}=X_{H}$, while $j^{2}=0$ implies $p_{Q}=p_{H}$ (contact discontinuity). In the Appendix we show how the last condition $\left(v_{H}=c_{s, H}\right)$ is solved as a function of $T$ and $\mu_{B}$ (or $T, \mu_{q}$, and $\mu_{s}$, when strangeness is included). We remark that the Chapman-Jouguet lines are essential for determining the region of physically allowed detonations and deflagrations. ${ }^{36}$

In Fig. 6 we show the influence of the parameters of our model equation of state on the physically allowed regions. These grow, when $B$ or $\Lambda$ is increased or when $C$ $(\mathcal{K})$ is decreased. Furthermore, in all cases the plasma energy density $\bar{e}_{Q}$, where the physically allowed region sets in $(\Delta \sigma=0)$, depends strongly on the entropy per net baryon $\sigma_{Q}$ in the initial (quark) phase. For given $\bar{e}_{H}$, this value of $\bar{e}_{Q}$ is smaller if $\sigma_{Q}$ is larger.

The position of the $(\Delta \sigma=0)$ line is an essential feature of the $\bar{e}_{Q}-\bar{e}_{H}$ diagrams in Fig. 6. Since the $\left(j^{2}=\infty\right)$, $\left(j^{2}=0\right),\left(v_{H}=c_{s, H}\right)$ curves do not shift appreciably in the region of physically allowed final states, it is this curve which determines the size of these regions. In general it is true that the larger the region is, the larger is the maximum possible hadronic entropy in this region. In Fig. 7 we illustrate this, displaying curves of $7 \%, 15 \%$, and $25 \%$ entropy increase compared to the initial (quark) entropy, ${ }^{51}$ calculated with a nonlinear version of the Walec$\mathrm{ka}$ model for the hadronic part of the nuclear equation of state. We mention that this equation of state yields quantitatively the same results as the other, phenomenological equations of state. This is due to the fact that the vector part of the potential energy in the meson field theory 
resembles strongly the interaction potential $U$, while the reduction of the effective nucleon mass has the same effect as adding resonances to the system.

We remark, however, that one cannot quantify the relation between the value of the maximum possible entropy and the size of the region by a simple linear proportionality. For instance, two completely different parame- ter sets $C, B, \Lambda, \sigma_{Q}$, which yield regions of physically allowed states of the same size, do not necessarily produce the same value for the maximum possible entropy in this region.

The influence of the new equation of state (11) is illustrated in Fig. 8. Since it is relatively soft (see above) compared to (10) in the energy density range of interest
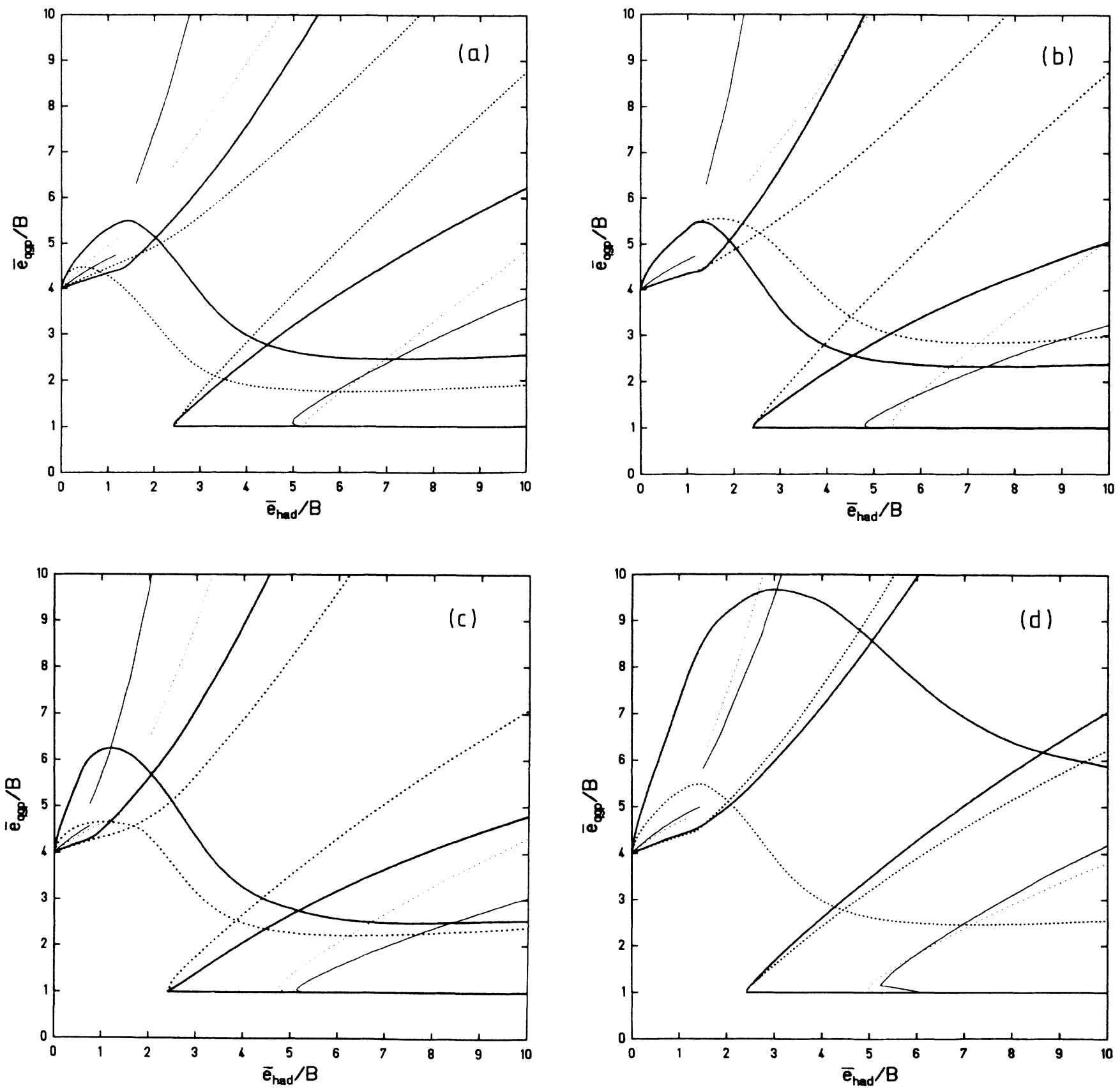

FIG. 6. The influence of varying the parameters $\sigma_{Q}, C, B$, and $\Lambda$ on the $\bar{e}_{Q}-\bar{e}_{H}$ diagram for equation of state (10). (a) $C=2650$ $\mathrm{MeV}, B=208 \mathrm{MeV} \mathrm{fm}^{-3}, \Lambda=0$ : dotted, $\sigma_{Q}=20$; solid, $\sigma_{Q}=10$. (b) $\sigma_{Q}=10, B=208 \mathrm{MeV} \mathrm{fm}^{-3}, \Lambda=0$ : dotted, $C=1200 \mathrm{MeV}$; solid, $C=3500 \mathrm{MeV}$. (c) $\sigma_{Q}=10 C=2650 \mathrm{MeV}, \Lambda=0$ : dotted, $B=140 \mathrm{MeV} \mathrm{fm}^{-3}$; solid, $B=400 \mathrm{MeV} \mathrm{fm}^{-3}$. (d) $\sigma_{Q}=10, C=2650 \mathrm{MeV}$, $B=208 \mathrm{MeV} \mathrm{fm}^{-3}$ : dotted, $\Lambda=0$ : solid, $\Lambda=200 \mathrm{MeV}$. No strange particles are considered. 


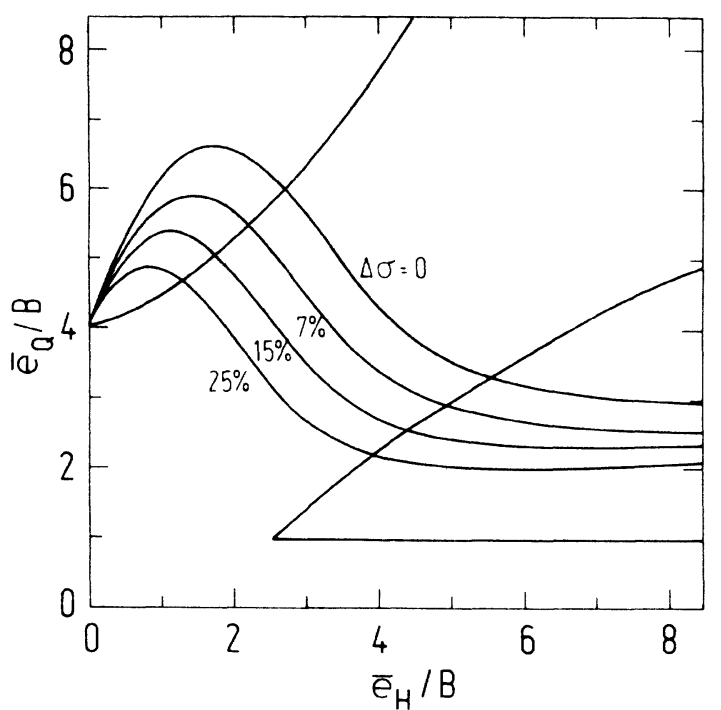

FIG. 7. Curves showing an entropy increase of $7 \%, 15 \%$, $25 \%$ for a calculation with $\sigma_{Q}=5, B=744 \mathrm{MeV} \mathrm{fm}^{-3}, \Lambda=0$ and with a nonlinear meson-field-theory equation of state $\left(K_{0}=300 \mathrm{MeV}, m_{0}^{*}=0.75, c_{V}=11.51\right)$.

(their stiffness can be made equal only for $x \rightarrow \infty$, while around the phase transition $[x \leq 25$, cf. Fig. 2(c)] this is not possible), the $(\Delta \sigma=0)$ curve is shifted upward at the same $\sigma_{Q}$ [cf. the influence in Fig. 6(b)]. This effect is enhanced further by the presence of strange particles; see Fig. 8. However, as mentioned above, this does not

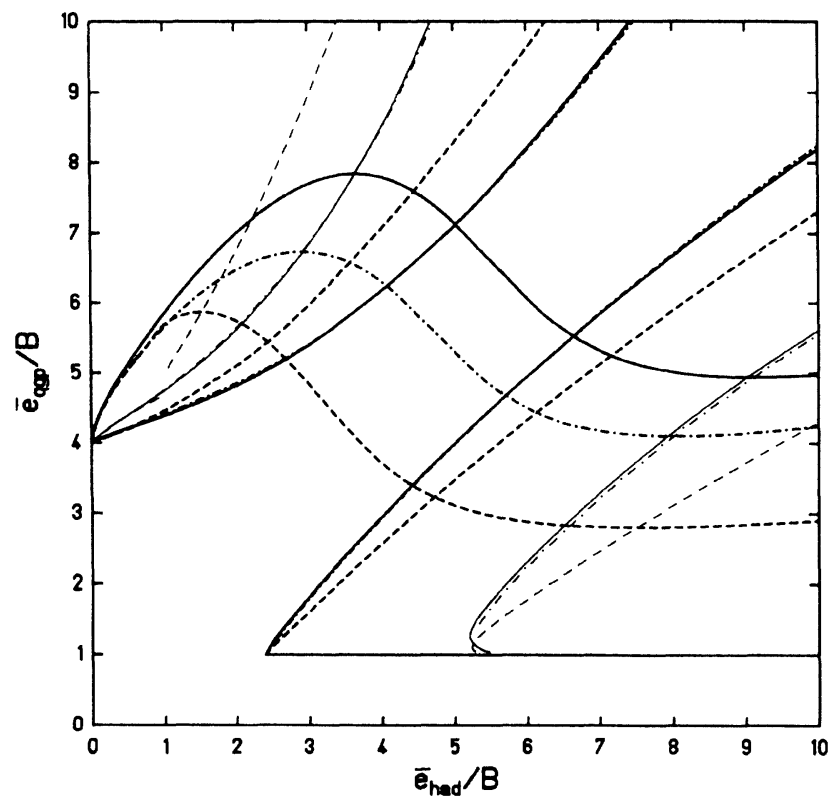

FIG. 8. Comparison between equations of state (10) and (11), with and without strangeness at the same asymptotic stiffness. Dashed line, (10) with $C=1800 \mathrm{MeV}$; the other lines, (11) with $\mathcal{K}=6.25$; solid line, with strange particles; dashed-dotted line, without strange particles. $B=250 \mathrm{MeV} \mathrm{fm}^{-3}, S / A=0$, $\sigma_{Q}=10$. necessarily imply that there is an appreciable increase in the maximum entropy production, compared to the calculation without strange particles or with equation of state (10). This can be settled only by an explicit calculation (see Sec. IV).

The physical relevance of a combustion shock is connected with the degree of supercooling of the plasma or superheating of hadronic matter, respectively. To determine the region in the $\bar{e}_{Q} \bar{e}_{H}$ diagram where the plasma is supercooled or hadron matter superheated, one calculates the energy density $\bar{e}_{Q}^{*}$ of quark matter at the intersection of the equilibrium phase boundary with the quark isentrope $\sigma_{Q}$ in the $T-\mu$ plane. Below this "critical" energy density $\frac{Q}{e}{ }_{Q}^{*}$ (arrow in Fig. 9) quark matter is supercooled. The question whether a final hadronic state is superheated or not is more complicated. If it is superheated, $\bar{e}_{H}$ exceeds the value $\bar{e}_{H}^{*}$, which corresponds to the intersection of the RHTA with the equilibrium phase boundary in the $T-\mu$ plane. It is obvious that this value depends on the respective RHTA and hence gives rise to the occurrence of a curve [rather than a straight line as for $\mu_{q}=0$ (Ref. 13)] in the $\bar{e}_{Q}-\bar{e}_{H}$ diagram (Fig. 9).

The result of this analysis is that almost all denotations and, under certain choices of the parameters, also deflagrations end in superheated hadronic matter. Detonations, however, require large hadronic energy densities or strong QGP supercooling, respectively, probably rendering these solutions physically irrelevant. On the other hand, deflagrations can start from either normal or supercooled QGP to end in normal (or superheated) hadron matter, depending on the position of the $(\Delta \sigma=0)$ curve. (In Fig. 9 all deflagrations require supercooled

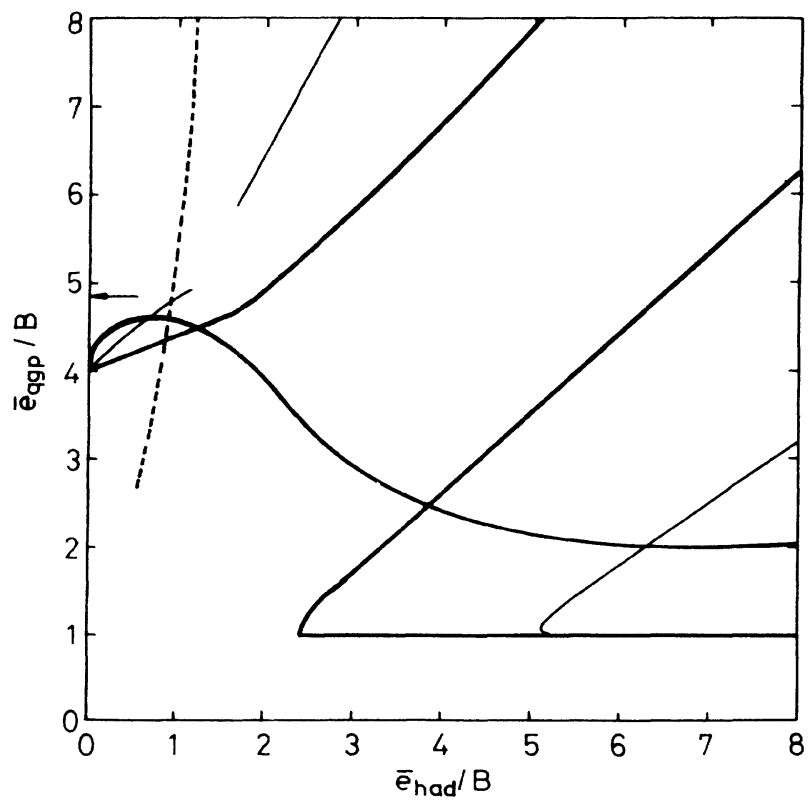

FIG. 9. Below the arrow quark matter is supercooled and to the right of the dashed curve hadronic matter is superheated; parameters as in Fig. 5. 
QGP. This, however, is due to the choice of the parameter set. For other sets, i.e., for larger values of $B, \Lambda$ and smaller values of $\sigma_{Q}$, also deflagrations from normal QGP are possible.)

\section{ENTROPY PRODUCTION AND CONSEQUENCES FOR PARTICLE RATIOS AT FREEZEOUT}

In this section we point out a possibility to check, by experimental means, the physical relevance of the above investigated shock discontinuities in relativistic heavy-ion collisions. We apply the general results of the last section to calculate the entropy production in a collision. In the above developed picture of the reaction there are two sources for entropy production: the compression shock discontinuity and, in case a plasma is created, the combustion discontinuity in which quark matter rehadronizes.

Let us first consider entropy production in a compression shock. In Fig. 10 we show [for the equation of state (10)] entropy production in single compression shocks from the ground state of nuclear matter into excited hadronic or quark matter (cf. also Refs. 8 and 9). The solution of the RHTA Eq. (14) is displayed in terms of the entropy per baryon $\sigma$ as a function of the bombarding energy (15). In order to give an idea of the beam energies necessary to produce a thermodynamically stable QGP, the region corresponding to equilibrium phase coexistence is also plotted [again using (15) to convert the energy density at the phase boundary into $\left.E_{\mathrm{lab}}^{\mathrm{kin}}\right]$.

Following the arguments about stability of shocks given in Sec. III, the part of the QGP RHTA to the left of $E_{\mathrm{lab}}^{\mathrm{kin}} \lesssim 15 \mathrm{GeV} /$ nucleon corresponds to unstable single shocks. However, when comparing entropy production in a hadronic and plasma scenario we are interested in larger bombarding energies. A detailed analysis of possible double shock formation would nevertheless be interesting, but is outside the scope of this paper.

Our main result is that the entropy in a pure hadronic scenario is determined by the parameter $C$ which controls the stiffness of the hadronic equation of state. ${ }^{9}$ While the entropy production for stiff equations of state is about $20 \%$ smaller than in the QGP scenario [Fig. 10(b)], there is almost no difference in entropy production for a soft equation of state [Fig. 10(a)]. Under the assumption that the nuclear equation of state is "stiff," this result is somewhat encouraging, since it indicates that in the case of QGP creation more entropy is produced than in a purely hadronic collision scenario. This leads to changes in the particle ratios at freezeout, which might, in turn, be used as a signal for plasma formation. We note, that preliminary WA80 data ${ }^{52}$ agree, in the order of magnitude, with our simple calculations. However, since we do not know which value of $C$ (or even which functional form for the hadronic equation of state) to choose, an unambiguous prediction of QGP creation through enhanced entropy production in the compression stage is not possible. This is confirmed when one calculates the entropy production with the equation of state (11) (Fig. 11). Compared to (10), it is relatively soft in the low baryon density region and a similar stiffness is obtained only in the limit
$\bar{\rho}_{B} \rightarrow \infty$. Hence, with respect to entropy production, this equation of state resembles a softer equation of state of type (10), cf. Fig. 10(a).

In Fig. 11 we also show the influence of strange particles. In general adding more particles to the system increases $\sigma$ at a given bombarding energy. This explains why similar calculations for the Walecka model, ${ }^{8}$ where only nucleons are considered, yield large differences in the entropy production between the plasma and the hadronic scenario. On the other hand, in our model, where in addition to the nucleons many resonances are included (cf. Table I), there is almost no difference. It is probably not realistic to assume that the heavier resonances can reach thermodynamical equilibrium in a heavy-ion collision. ${ }^{53}$ However, under the assumption that all resonances are equilibrated, we obtain, according to the
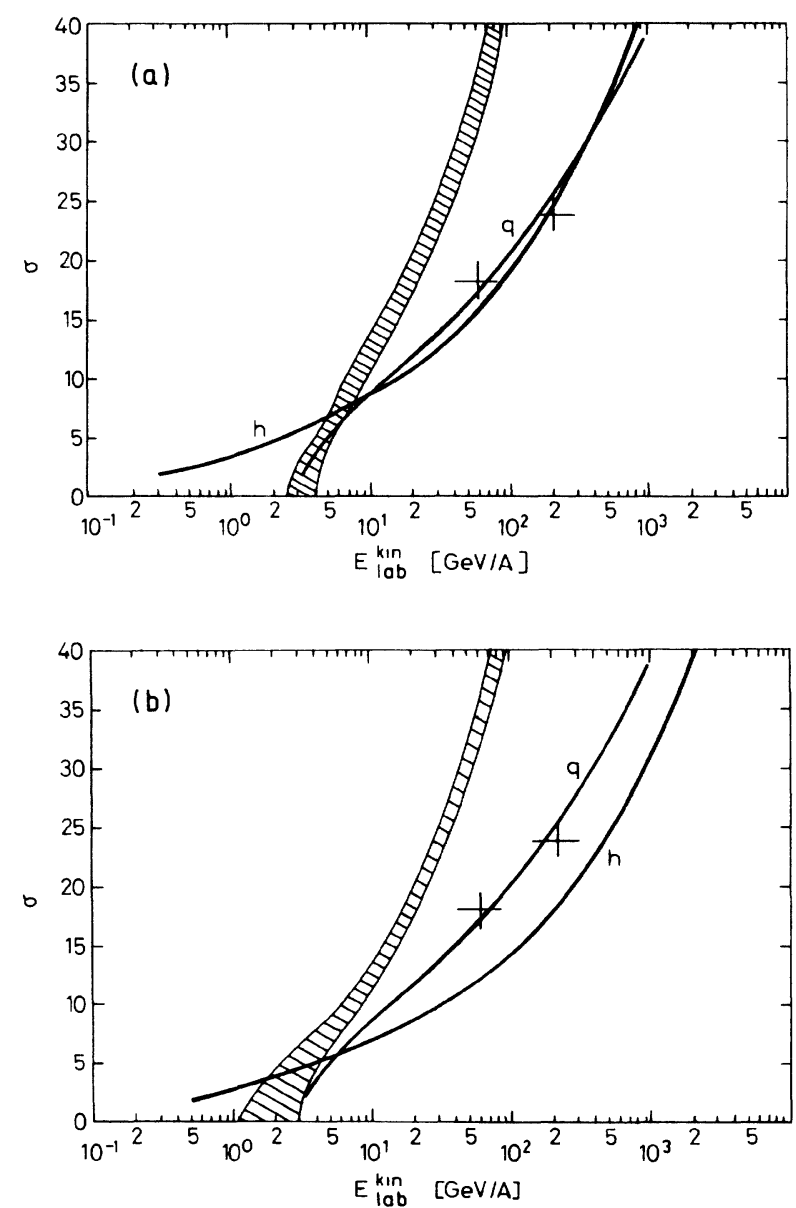

FIG. 10. RHTA's for hadronic and quark matter as excitation functions of the entropy per baryon for (a) a soft equation of state (10), $C=1400 \mathrm{MeV}$, and (b) a stiff equation of state, $C=3500 \mathrm{MeV}$. In both cases no strange particles are considered, $B=250 \mathrm{MeV} \mathrm{fm}^{-3}, \Lambda=100 \mathrm{MeV}$. For orientation also the phase coexistence region is depicted (hatched area). Crosses correspond to WA80 data (Ref. 52) at CERN's $60-\mathrm{GeV} /$ nucleon and $200-\mathrm{GeV} /$ nucleon experiments. 


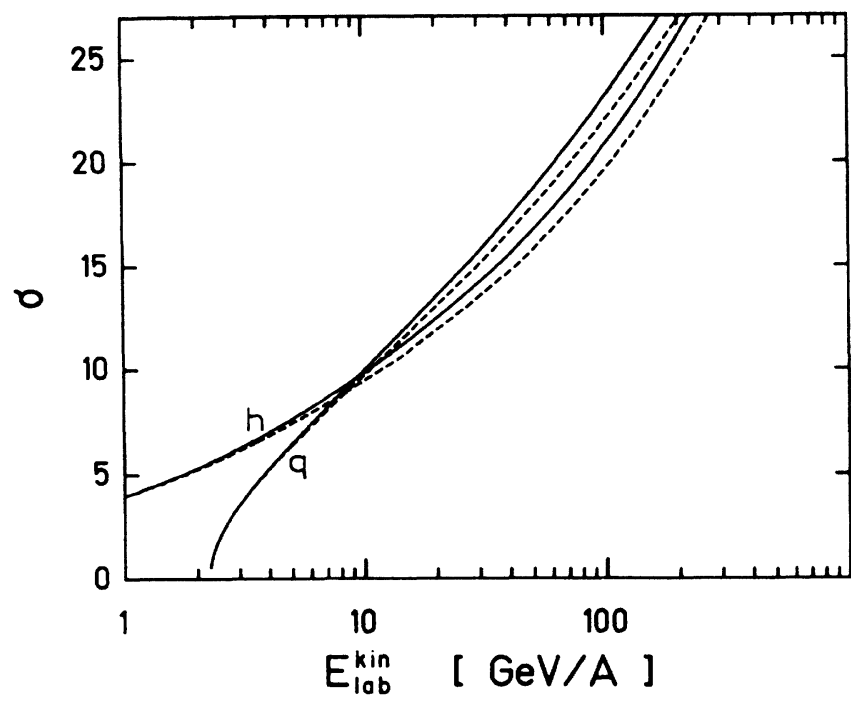

FIG. 11. RHTA's for hadronic and quark matter as excitation functions of the entropy per baryon for equation of state (11), $\mathcal{K}=6.25, B=250 \mathrm{MeV} \mathrm{fm}^{-3}$. Solid lines for a calculation including strange particles, dashed lines for a completely nonstrange scenario.

above arguments, an upper limit for the entropy produced in the hadronic scenario.

If one considers the compression stage alone, one cannot resolve the question whether entropy production is enhanced in the case of QGP creation. However, there is also the possibility that entropy is produced via the second source mentioned above: a combustion (deflagration or detonation) shock. ${ }^{54}$ This additional entropy increase might be sufficient to lead to a significant, i.e., observable change in an experimentally measurable quantity. For vanishing net baryon density the entropy increase does not exceed $7 \%$ (Ref. 15). For a baryon-rich scenario, however, this may be considerably larger.

In Sec. III we have shown that there are physically allowed combustion processes in the phase transition from QGP to hadrons. The main problem was, however, to precisely determine the final hadronic state after the combustion shock. This is in general not possible, since, as mentioned above, one does not know (a) at which $T_{Q}$ and $\sigma_{Q}$ the combustion sets in and (b) where on the corresponding RHTA it ends. We consider at first only the nonstrange scenario. As a first step we calculate the Taub adiabat with a fixed center $T_{Q}, \sigma_{Q}$ for a certain choice of parameters $C, B, \Lambda$. By inspection of the RHTA we then find the final shock state, which most probably corresponds to maximum entropy production (the deflagration $C J$ point). We then vary $T_{Q}, \sigma_{Q}$ over a plausible range and calculate the entropy produced in such a final shock state.

Concerning (a), let us first fix the parameters of our model. We choose the values $C=2650 \mathrm{MeV}, B=400$ $\mathrm{MeV} \mathrm{fm}^{-3}$ and, for the sake of simplicity, $\Lambda=0 \mathrm{MeV}$. We then assume that the combustion sets in on the quark isentrope $\sigma_{Q}=25$, which, in our compression model, corresponds to $E_{\text {lab }}^{\text {kin }}=200 \mathrm{GeV} /$ nucleon, using the same pa- rameters. The temperature is $T_{Q}=160 \mathrm{MeV}$, which corresponds to a supercooling of the QGP by $\Delta T \simeq 10 \mathrm{MeV}$ below the equilibrium phase boundary along the isentrope. Note that, since we do not know the hadronization time scale, this choice of $\Delta T$ is almost completely arbitrary. The above value is of the order of magnitude of the value that is obtained under the reasonable assumption that the plasma survives for $\delta t \sim \Lambda^{-1} \sim 1 \mathrm{fm}$, after passing the equilibrium phase boundary in a threedimensional isentropic expansion.

To answer (b) we now calculate the RHTA corresponding to $\left(\sigma_{Q}, T_{Q}\right)=(25,160 \mathrm{MeV})$. We display the results in a $\sigma_{H}-\bar{e}_{H}$ diagram (Fig. 12) and observe that physically possible detonations require unreasonably large energy densities in the final hadron state, as was surmised above (note the log scale in Fig. 12). Hence we assume that the hadronization takes place in a deflagration front. In such a process the maximum entropy is produced at the Chapman-Jouguet point: $\sigma_{H}=28.9$, i.e., an increase of $\Delta \sigma=3.9 \simeq 15 \%$ compared to an adiabatic expansion of the QGP.

We remark that the general property of the CJ points, namely, that they correspond to a local maximum of the entropy for deflagrations and to a local minimum for detonations, ${ }^{36}$ is nicely confirmed in Fig. 12. This is also directly related to the fact that the CJ lines in Figs. 5, 6, and 8 pass the local maxima and minima of the $(\Delta \sigma=0)$ curves. Furthermore we note, that for the scenario of Fig. 12 the maximum entropy produced in the hadronization phase transition is independent of whether the process is a deflagration or a condensation ${ }^{36}$ (cf. Ref. 54).

We now investigate the entropy production in

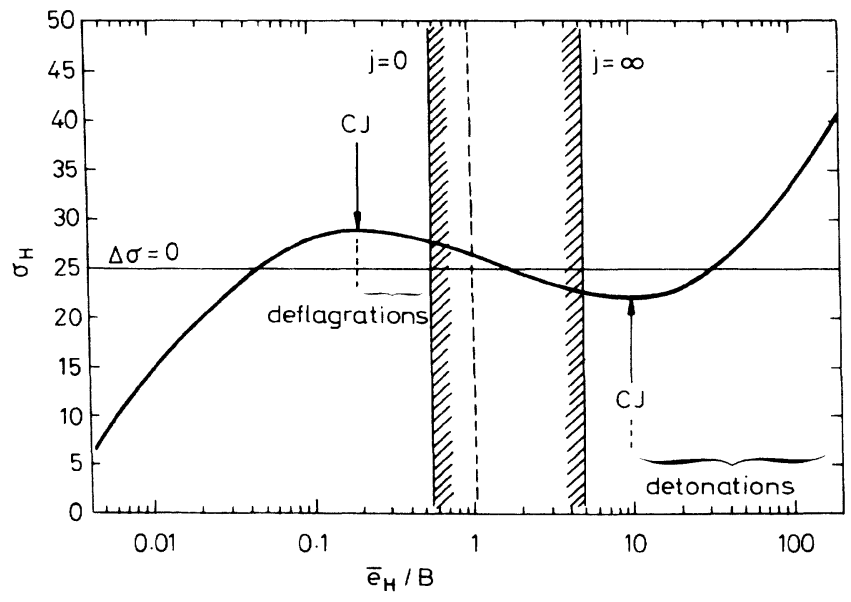

FIG. 12. $\sigma_{H}-\bar{e}_{H}$ diagram of a particular RHTA, $\sigma_{Q}=25$, $T_{Q}=160 \mathrm{MeV}, C=2650 \mathrm{MeV}, B=400 \mathrm{MeV} \mathrm{fm}^{-3}, \Lambda=0$, without strange degrees of freedom. Below the horizontal line $(\Delta \sigma=0)$ shocks are forbidden, because the condition of entropy increase across the shock front is violated. Hatched lines demarcate physically forbidden shocks with $j^{2}<0$. To the right of the dashed line hadron matter is superheated. The Chapman-Jouguet points are marked with CJ. The conditions of mechanical stability, (IIIa) and (IIIb) of Eq. (20), yield the physically allowed regions of spacelike deflagrations and detonations. 
Chapman-Jouguet deflagration shocks for various initial states $\left(T_{Q}, \sigma_{Q}\right)$. A reasonable choice for $\left(T_{Q}, \sigma_{Q}\right)$ are their values along the phase boundary. ${ }^{14,41}$ However, since we also want to explore the effects of supercooling we choose $T_{Q}, \sigma_{Q}$ according to the following procedure.

(a) Estimate the initially produced entropy per baryon $\sigma_{Q}$ via a compression shock calculation for given $E_{\text {lab. }}^{\mathrm{kin}}$.

(b) Expand the system isentropically according to the time evolution law ${ }^{47}$

$$
\frac{\bar{\rho}_{B}(t)}{\bar{\rho}_{B}(t=0)}=\left(1+\frac{t}{t_{0}}\right)^{-n},
$$

where $n=1,3$ for longitudinal or spherical expansion, respectively. The expansion starts at $t=0$. The value of $t_{0}$ is assumed to be $5 \mathrm{fm}$, which corresponds roughly to the time scale of the hydrodynamic expansion of a sphere of radius $3 \mathrm{fm}$.

(c) Calculate the time $t^{\mathrm{PT}}$, when the phase boundary is reached,

$$
t^{\mathrm{PT}}=t_{0}\left[\left(\frac{\bar{\rho}_{B}(t=0)}{\bar{\rho}_{B}^{\mathrm{PT}}}\right]^{1 / n}-1\right],
$$

where $\bar{\rho}_{B}^{\mathrm{PT}}$, for a given $\sigma_{Q}$, is obtained from the Gibbs conditions for phase equilibrium.

(d) Calculate the density $\bar{\rho}_{B}\left(t^{\mathrm{PT}}+\delta t\right)$ using Eq. (21) after a time interval $\delta t \simeq \Lambda^{-1}$ during which the plasma supercools.

(e) Use the corresponding values of $T_{Q}$ and $\sigma_{Q}$ as initial state for the deflagration shock leading to the $C J$ point of the respective Taub adiabat.

The results are displayed in Fig. 13. In Fig. 13(a) we show the centers of the Taub adiabats in the quark phase and the final $(\mathrm{CJ})$ points in the hadron phase for $\delta t=0,1$, $1.5 \mathrm{fm}$. Note that for $\delta t=0$ the deflagration shock starts from the phase boundary. For $\delta t \geq 1 \mathrm{fm}$ the plasma is supercooled far into the region of stable hadronic matter. A shock starting from the phase boundary may create superheated hadronic matter while a moderately supercooled plasma in most cases decays into stable hadronic matter. One observes [Fig. 13(b)] that the entropy increase is small for $\delta t<1 \mathrm{fm}$ and that the values of the relative entropy increase agree roughly with those obtained in Ref. 14. Note that shocks with $\sigma_{H}^{\mathrm{CJ}} / \sigma_{Q}<1$ are physically not allowed. The actual values of $\sigma_{Q}$ at which this happens depend on the choice of the parameters of our model [cf. Fig. 13(c)].

To understand the fact there are no solutions beyond a certain value of $\sigma_{Q}$ for $\delta t=1,1.5 \mathrm{fm}$ in Fig. 13(b) it is useful to remember that along the phase transition line $\bar{e}$ diminishes with increasing $\sigma$ (Refs. 9 and 21). This means that the phase transition value of $\bar{e}_{Q}$ (arrow in Fig. 9) approaches the value $4 B$, as $\sigma_{Q}$ increases. Then the energy density $\bar{e}_{Q}\left(t^{\mathrm{PT}}+\delta t\right)$ (of the initial shock state) can even be smaller than $4 B$, which means that deflagrations are not possible (Fig. 9). However, detonation shocks are still allowed. It is clear that for stronger supercooling $(\delta t=1.5 \mathrm{fm})$ this phenomenon occurs at smaller values of $\sigma_{Q}$ [cf. Fig. 13(b)]. In Fig. 13(c) we show the influence of varying the parameters of our model on the entropy production for $\delta t=1 \mathrm{fm}$ supercooling. The entropy produc-
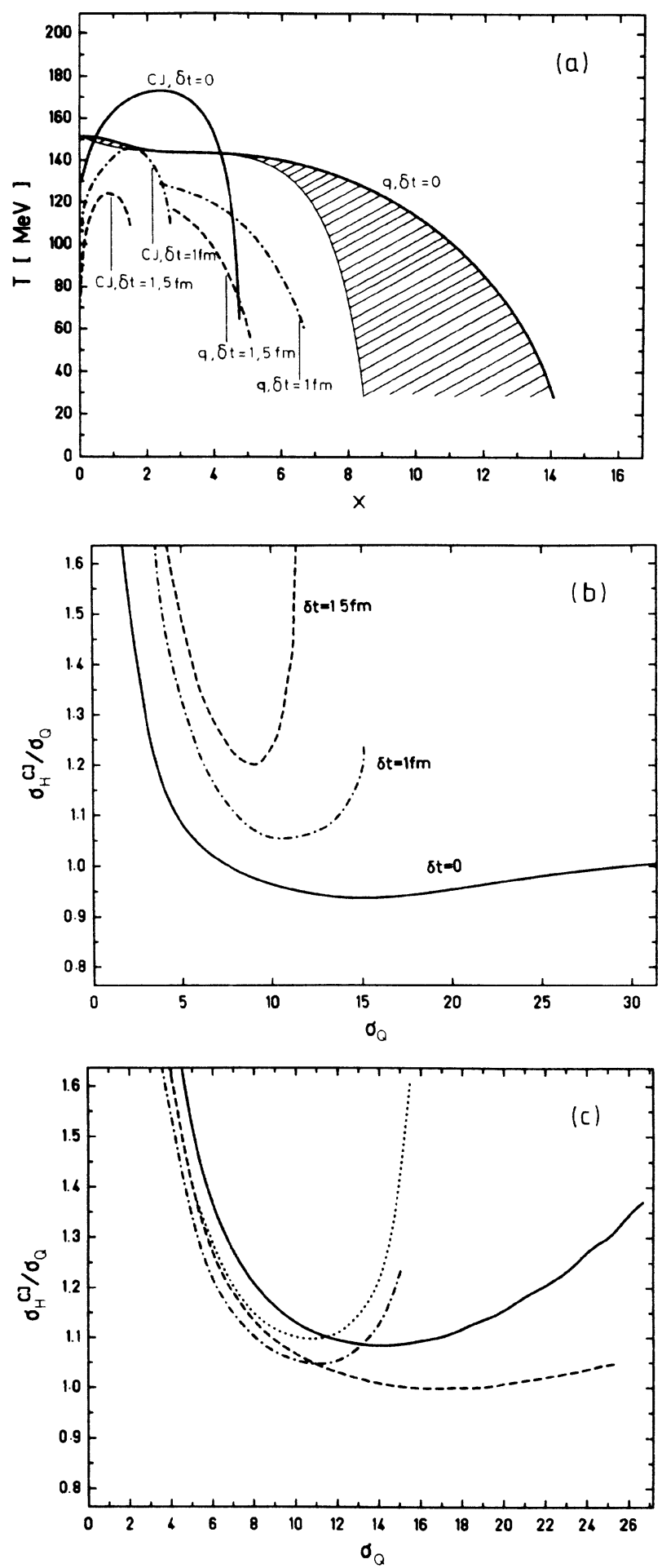

FIG. 13. T-x diagram indicating Chapman-Jouguet (CJ) deflagrations starting from the phase boundary and from supercooled QGP states (marked with $q$ ). Solid line, $\delta t=0$, dasheddotted line, $\delta t=1 \mathrm{fm}$; dashed line, $\delta t=1.5 \mathrm{fm}$ supercooling time. The parameters are $B=250 \mathrm{MeV} \mathrm{fm}^{-3}, \Lambda=0, \mathcal{K}=6.25$, $n=3$. (b) Relative entropy excess gained via deflagrations corresponding to Fig. 13(a). (c) Influence of the parameters of our model on the entropy production after $1 \mathrm{fm}$ supercooling. Dashed-dotted line, parameters as in Fig. 13(b). Solid line, as in (b) but for $B=400 \mathrm{MeV} \mathrm{fm}^{-3}$; dashed line, as in (b) but for $\Lambda=100 \mathrm{MeV}$; dotted line, as in (b) but for $\mathcal{K}=12.25$. 
tion at small $\sigma_{Q}$ is fairly independent of the parameters, while the region in $\sigma_{Q}$ where deflagrations are allowed depends strongly on $B$ and $\Lambda$.

We now discuss the effect of the entropy excess obtained in our simple model on the final hadronic particle ratios in a heavy-ion collision. We make the simple assumption that the system freezes out below a certain baryon density $\bar{\rho}_{B}=\rho_{\mathrm{FO}}$, e.g., $0.02 \mathrm{fm}^{-3}$. In principle, rather than the net baryon density, one should use the total particle density since it can be directly related to the mean free path of the particles, which in turn gives a criterion for decoupling if it exceeds the size of the system. However, in the cases of interest to us $\bar{\rho}_{B} \simeq \rho^{\text {tot }}=\sum_{i} \bar{\rho}_{i}$ is a good approximation. We then calculate inclusive particle ratios (i.e., particle ratios after decay of resonances) for a given value of the specific entropy $\sigma_{H}$ at $\bar{\rho}_{B}=\rho_{\mathrm{FO}}$, obtained under the assumption of thermodynamical equilibrium.

For illustrative purposes we first return to the scenario of Fig. 12, where we get a total entropy increase of about $25 \%$ compared to a purely hadronic scenario. Approximately half of the gain in entropy is produced in the compression stage (for this choice of parameters). We have for the inclusive antinucleon-to-nucleon ratio in the "pure" hadronic case

$$
\sigma_{H}=21 \rightarrow \frac{\langle\bar{N}\rangle}{\langle N\rangle}=7.36 \times 10^{-4},
$$

and in the case that a QGP is created which decays spontaneously into hadrons

$$
\sigma_{H}=28.9 \rightarrow \frac{\langle\bar{N}\rangle}{\langle N\rangle}=7.72 \times 10^{-3} .
$$

That an increase of about $25 \%$ in $\sigma$ results in order-ofmagnitude effects is most easily understood by noting that a change of $\sigma$ implies a change of $\mu / T$ of the same order (cf. the nonrelativistic Boltzmann gas case: $\left.\sigma \sim \frac{5}{2}-\mu / T\right)$, but that antiparticle-to-particle ratios vary exponentially with changes of $\mu / T$ [cf. the Boltzmann approximation of $\left.\langle\bar{N}\rangle /\langle N\rangle \sim \exp \left(-2 \mu_{N} / T\right)\right]$. Therefore differences in $\sigma$ are exponentiated by antiparticle-toparticle ratios.

In Fig. 14 we show inclusive particle ratios as a function of $\sigma_{H}$ at $\rho_{\mathrm{FO}}=0.02 \mathrm{fm}^{-3}$ for the equation of state (11), including strange particles. As anticipated above, the ratios $\langle\bar{N}\rangle /\langle N\rangle,\langle\bar{\Lambda}\rangle /\langle\Lambda\rangle$ are promising candidates for detecting an enhancement in the entropy production if a QGP is created; these ratios vary by about an order of magnitude if the entropy is altered by only 5 units. The ratio $\langle\bar{N}\rangle /\langle\pi\rangle$ shows a behavior similar to that of the antiparticle-to-particle ratios, due to the fact that $-\left(m_{\bar{N}}+\tilde{\mu}_{B}\right) / T$ increases more rapidly with increasing $\sigma$ than $-m_{\pi} / T$ along $\bar{\rho}_{B}=0.02 \mathrm{fm}^{-3}=$ const.

We note that $\langle K\rangle /\langle\pi\rangle$ and $\langle\bar{K}\rangle /\langle\pi\rangle$ have only a weak dependence on the entropy in the range of interest and are therefore not significantly affected by excess entropy production in the case of plasma creation. The ratio $\langle K\rangle /\langle\bar{K}\rangle\left(\sim \exp \left[+2\left(\mu_{q}-\mu_{s}\right) / T\right]\right)$ shows an interesting behavior; the dependence on $\sigma$ is inversed, compared to the other ratios. The reason is that for small $\sigma$ $\mu_{q}-\mu_{s}$ is positive and large, while for large $\sigma$ it nearly vanishes along $\bar{\rho}_{B}=$ const. Hence the ratio approaches the ratio of the corresponding degeneracies $g_{K} / g_{\bar{K}}=1$. A similar behavior was found in Ref. 24 .

We remark that all ratios are independent of the value of $\mathcal{K}$ in the representation chosen in Fig. 14, since the equation of state (8) is constructed such that $\sigma_{H}$ and $\bar{\rho}_{i}$ depend only on $T$ and $\bar{\rho}_{B}$, and not explicitly on $\mathcal{K}$ (Ref. 9).

To check the dependence of our results on $\rho_{\text {FO }}$ we recalculated the ratios for $\rho_{\mathrm{FO}}=0.08 \mathrm{fm}^{-3}$. Although the absolute values of the ratios are up to an order of magnitude larger, the essential assertion, that the difference be-
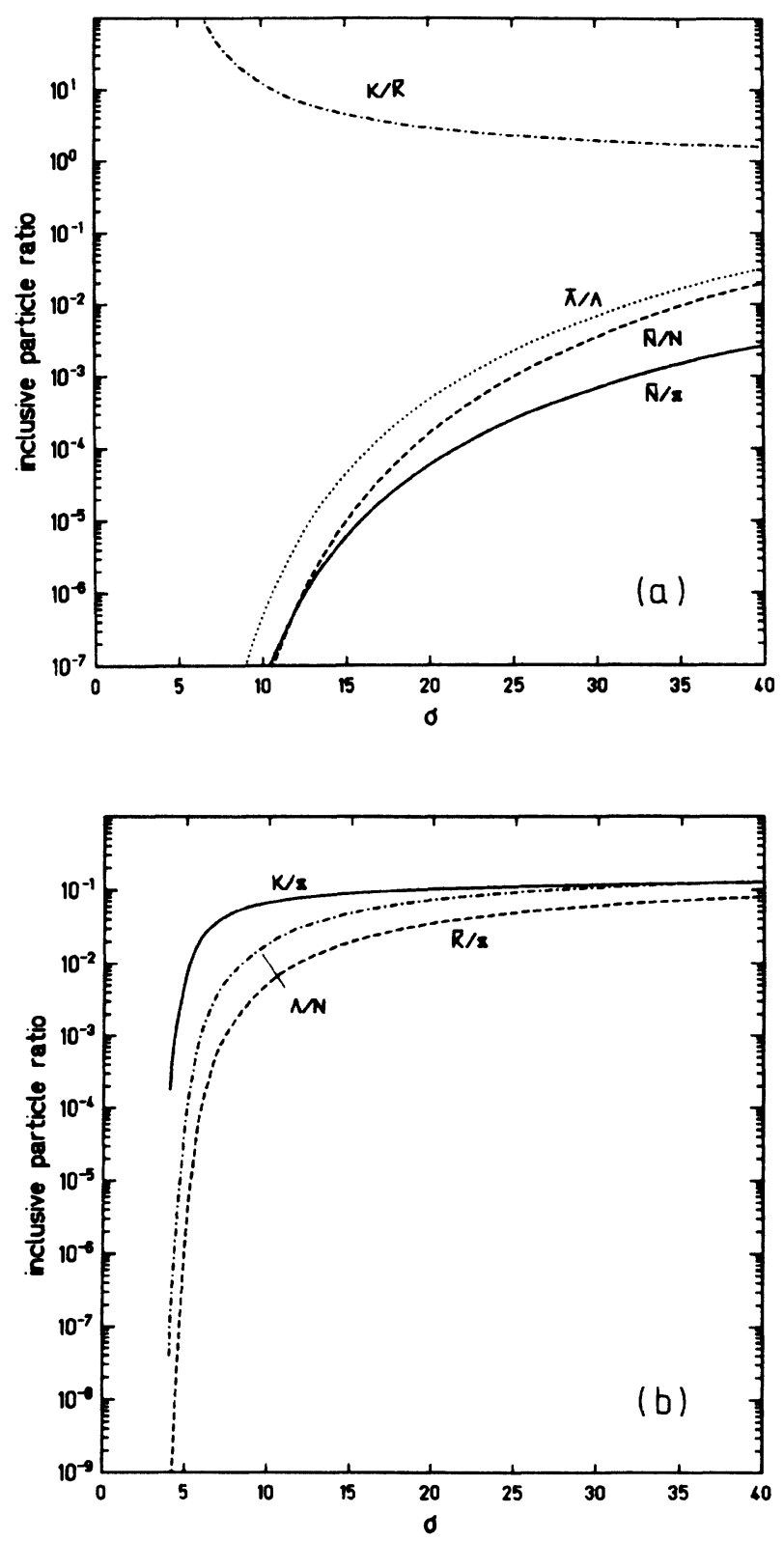

FIG. 14. Inclusive particle ratios at freezeout $\left(\rho_{\mathrm{FO}}=0.02\right.$ $\mathrm{fm}^{-3}$ ) for equation of state (11). 
tween ratios for entropies differing by $\sim 5$ units may be $\sim 1$ order of magnitude, remains valid. The absolute values should in any case not be taken too seriously because of the assumptions on the parameters and on the dynamical evolution of our system. One cannot expect the final particle ratios to be insensitive to these assumptions. Furthermore the influence of a more realistic freezeout model ${ }^{56,57}$ and of nonequilibrium processes (especially of chemical nature $)^{24,53,58}$ should be investigated.

The point, however, is that, in the case of QGP creation and rehadronization through a combustion discontinuity, an appreciable increase of particle ratios relative to a hadronic collision scenario could occur. It would be especially helpful, if an excitation function of these ratios becomes available by experiment. The creation of a QGP would then be identified by a strong increase in the above-mentioned ratios at a certain c.m. energy density, which indicates the deconfinement transition.

In conclusion we would first like to remark that the effect of enhanced antimatter production could be even more dramatic than in our illustrative example, since the increase in entropy may exceed $25 \%$ [cf. Figs. 13(b) and 13(c)]. Furthermore, we note that a combustion shock is not necessarily a global phenomenon. It could occur locally, i.e., in certain rapidity intervals. One should thus look for correlations between fluctuations in the $d N / d y$ distribution and enhanced particle ratios per rapidity interval. Finally, considering only relative enhancements of the entropy per baryon and per unit rapidity and relative enhancements of the antiparticle abundancies would remove the dependence on quantitative details of our simple model of a relativistic heavy-ion collision.

\section{CONCLUSIONS AND SUMMARY}

In this paper we have studied compression shocks and spacelike hadronizing combustion discontinuities of a quark-gluon plasma in baryon-rich nuclear matter. The quantum statistics of the constituents were consistently taken into account and strange degrees of freedom were included. We have proposed a new phenomenological formula for the compressional energy of hadronic matter to avoid shortcomings of an older ansatz. Both of these forms, as well as a nonlinear meson field theory, are applied as a hadronic equation of state in the calculation of shock discontinuities. We find that the results of the shock calculations are not qualitatively affected by the particular form of the hadronic equation of state. On the other hand, the stability of compression shocks into the mixed phase depends on the stiffness of the equation of state.

As an application we have calculated the entropy produced in a shock model of a heavy-ion collision. The entropy production in the compression stage is not significantly larger in a plasma scenario as compared to a purely hadronic collision scenario. However, if the phase transition between quarks and hadrons takes place in a combustion (i.e., deflagration) shock, starting from a supercooled QGP, excess entropy is created, which may drastically alter the chemical composition of the system at freezeout. We found a strong dependence of antiparticle-to-particle ratios on the final-state entropy. Thus, in the case that a quark-gluon plasma is created, enhancement of antiparticle-to-particle ratios by an order of magnitude or perhaps even more may be observable.

\section{ACKNOWLEDGMENTS}

We thank H. W. Barz, C. H. Greiner, B. Kämpfer, J. Knoll, L. Neise, and H. Schulz for valuable discussions. This work was supported by the German Federal Minister of Research and Technology (BMFT) under Contract No. 060F772, by the Deutsche Forschungsgemeinschaft (DFG) under Contract No. GR 243/32-2 and by the Gesellschaft für Schwerionenforschung (GSI).

\section{APPENDIX A: THE VELOCITY OF SOUND (NONSTRANGE CASE)}

The aim is to express the velocity of sound in terms of $\mu$ and $T$. We have, using the functional determinant formalism (we omit mean bars and indices)

$$
c_{s}^{2}=\left[\frac{\partial p}{\partial e}\right]_{\sigma}=\frac{\partial(p, \sigma)}{\partial(T, \mu)}\left[\frac{\partial(e, \sigma)}{\partial(s, \rho)} \frac{\partial(s, \rho)}{\partial(T, \mu)}\right]^{-1} .
$$

The particular determinants are easily calculated with the help of the first law of thermodynamics,

$$
\begin{aligned}
d p=s d T & +\rho d \mu, \\
d e=T d s+\mu d \rho, & \left.+\left[\frac{\partial s}{\partial \mu}\right]_{T}-\sigma\left(\frac{\partial \rho}{\partial \mu}\right]_{T}\right] \\
\frac{\partial(p, \sigma)}{\partial(T, \mu)}=\sigma & -\left[\frac{\partial s}{\partial T}\right]_{\mu}+\sigma\left[\frac{\partial \rho}{\partial T}\right]_{\mu}, \\
\frac{\partial(e, \sigma)}{\partial(s, \rho)}= & -X, \\
\frac{\partial(s, \rho)}{\partial(T, \mu)}= & {\left[\frac{\partial s}{\partial T}\right]_{\mu}\left(\frac{\partial \rho}{\partial \mu}\right]_{T}-\left[\frac{\partial \rho}{\partial T}\right]_{\mu}\left(\frac{\partial s}{\partial \mu}\right)_{T}, }
\end{aligned}
$$

yielding, with the help of Maxwell's relations,

$c_{s}^{2}=\frac{1}{X} \frac{2 \sigma(\partial \rho / \partial T)_{\mu}-(\partial s / \partial T)_{\mu}-\sigma^{2}(\partial \rho / \partial \mu)_{T}}{\left[(\partial \rho / \partial T)_{\mu}\right]^{2}-(\partial s / \partial T)_{\mu}(\partial \rho / \partial \mu)_{T}}$.

The partial derivatives can now be calculated (since $\rho$ and $s$ are explicitly given as functions of $\mu$ and $T$ ):

$$
\begin{aligned}
& \left(\frac{\partial \rho}{\partial T}\right]_{\mu}=\frac{g(\mu, T)}{1+u^{\prime \prime} f(\mu, T)}, \\
& {\left[\frac{\partial \rho}{\partial \mu}\right]_{T}=\frac{f(\mu, T)}{1+u^{\prime \prime} f(\mu, t)},} \\
& \left(\frac{\partial s}{\partial T}\right]_{\mu}=h(\mu, T)-\frac{u^{\prime \prime} g^{2}(\mu, T)}{1+u^{\prime \prime} f(\mu, T)} .
\end{aligned}
$$

$u^{\prime \prime}$ is the second derivative of the mean potential energy 
density $\bar{u}$. The functions $f, g, h$, given by

$f=\sum_{i} \frac{g_{i} b_{i}^{2}}{2 \pi^{2} T} \int_{0}^{\infty} d k k^{2}\left(\bar{n}_{k}^{i}\right)^{2} \exp (\beta A)$,

$g=\sum_{i} \frac{g_{i} b_{i}}{2 \pi^{2} T} \int_{0}^{\infty} d k k^{2} \beta A\left(\bar{n}_{k}^{i}\right)^{2} \exp (\beta A)$,

$h=\sum_{i} \frac{g_{i}}{2 \pi^{2} T} \int_{0}^{\infty} d k k^{2}(\beta A)^{2}\left(\bar{n}_{k}^{i}\right)^{2} \exp (\beta A)$,

$\bar{n}_{k}^{i}=\left[\exp (\beta A)+\theta_{i}\right]^{-1}$

$A=\sqrt{k^{2}+m_{i}^{2}}-b_{i}(\mu-\Sigma)$

have to be integrated numerically.
APPENDIX B: THE VELOCITY OF SOUND (STRANGE CASE)

In the strange case the calculation is by far more complex. Let us now define $\sigma=s / \rho_{q}, X=(e+p) / \rho_{q}^{2}$, $f_{s}=\rho_{s} / \rho_{q}$. Then

$$
\begin{aligned}
c_{s}^{2} & =\left[\frac{\partial p}{\partial e}\right]_{\sigma, f_{s}} \\
& =\frac{\partial\left(p, \sigma, f_{s}\right)}{\partial\left(T, \mu_{q}, \mu_{s}\right)}\left[\frac{\partial\left(e, \sigma, f_{s}\right)}{\partial\left(s, \rho_{q}, \rho_{s}\right)} \frac{\partial\left(s, \rho_{q}, \rho_{s}\right)}{\partial\left(T, \mu_{q}, \mu_{s}\right)}\right]^{-1} .
\end{aligned}
$$

The determinants can be calculated, again using Maxwell's relations and the first law of thermodynamics,

$$
\begin{aligned}
& d p=s d T+\rho_{q} d \mu_{q}+\rho_{s} d \mu_{s} \\
& d e=T d s+\mu_{q} d \rho_{q}+\mu_{s} d \rho_{s},
\end{aligned}
$$

to yield

$$
\begin{aligned}
c_{s}^{2}= & \left\{2 \sigma\left[\frac{\partial \rho_{q}}{\partial T} \frac{\partial \rho_{s}}{\partial \mu_{s}}-\frac{\partial \rho_{q}}{\partial \mu_{s}} \frac{\partial \rho_{s}}{\partial T}\right]+2 \sigma f_{s}\left[\frac{\partial \rho_{s}}{\partial T} \frac{\partial \rho_{q}}{\partial \mu_{q}}-\frac{\partial \rho_{q}}{\partial T} \frac{\partial \rho_{q}}{\partial \mu_{s}}\right]+\sigma^{2}\left[\left(\frac{\partial \rho_{q}}{\partial \mu_{s}}\right)^{2}-\frac{\partial \rho_{q}}{\partial \mu_{q}} \frac{\partial \rho_{s}}{\partial \mu_{s}}\right]\right. \\
& \left.-\left[\frac{\partial s}{\partial T} \frac{\partial \rho_{s}}{\partial \mu_{s}}-\left[\frac{\partial \rho_{s}}{\partial T}\right]^{2}\right]+2 f_{s}\left[\frac{\partial s}{\partial T} \frac{\partial \rho_{q}}{\partial \mu_{s}}-\frac{\partial \rho_{q}}{\partial T} \frac{\partial \rho_{s}}{\partial T}\right]+f_{s}^{2}\left[\left(\frac{\partial \rho_{q}}{\partial T}\right]^{2}-\frac{\partial \rho_{q}}{\partial \mu_{q}} \frac{\partial s}{\partial T}\right]\right\} \\
& \times\left[-X\left\{\frac{\partial s}{\partial T}\left[\frac{\partial \rho_{q}}{\partial \mu_{q}} \frac{\partial \rho_{s}}{\partial \mu_{s}}-\left(\frac{\partial \rho_{q}}{\partial \mu_{s}}\right]^{2}\right]-\frac{\partial \rho_{q}}{\partial T}\left[\frac{\partial \rho_{q}}{\partial T} \frac{\partial \rho_{s}}{\partial \mu_{s}}-\frac{\partial \rho_{s}}{\partial T} \frac{\partial \rho_{q}}{\partial \mu_{s}}\right]+\frac{\partial \rho_{s}}{\partial T}\left[\frac{\partial \rho_{q}}{\partial T} \frac{\partial \rho_{q}}{\partial \mu_{s}}-\frac{\partial \rho_{s}}{\partial T} \frac{\partial \rho_{q}}{\partial \mu_{q}}\right]\right\}\right]^{-1} .
\end{aligned}
$$

For sake of simplicity we simply wrote, e.g., $\partial \rho_{q} / \partial T$ for $\left(\partial \rho_{q} / \partial T\right)_{\mu_{q}, \mu_{s}}$. In the limit $f_{s} \rightarrow 0, \partial \rho_{s} / \partial \mu_{s} \rightarrow 1$ we regain the result of Appendix A.

The explicit calculation of the various partial derivatives is similar to Appendix A, but quite more laborious. One has

$$
\begin{aligned}
& \frac{\partial \rho_{q, s}}{\partial T}=\left(1+u^{\prime \prime} \sum_{i} b_{i}^{2} f_{i}\right]^{-1}\left[\sum_{i} z_{q, s}^{i} e_{i}+\frac{u^{\prime \prime}}{3}\left(\sum_{i} z_{q, s}^{i} e_{i} \sum_{j} z_{s, q}^{j} b_{j} f_{j}-\sum_{i} z_{s, q}^{i} e_{i} \sum_{j} z_{q, s}^{j} b_{j} f_{j}\right]\right] \text {, } \\
& \frac{\partial \rho_{q}}{\partial \mu_{q}}=\left(1+u^{\prime \prime} \sum_{i} b_{i}^{2} f_{i}\right]^{-1}\left\{\sum_{i}\left(z_{q}^{i}\right)^{2} f_{i}+\frac{u^{\prime \prime}}{9}\left[\sum_{i}\left(z_{q}^{i}\right)^{2} f_{i} \sum_{j}\left(z_{s}^{j}\right)^{2} f_{j}-\left(\sum_{i} z_{q}^{l} z_{s}^{i} f_{i}\right]^{2}\right]\right\} \text {, } \\
& \frac{\partial \rho_{s}}{\partial \mu_{s}}=\left(1+u^{\prime \prime} \sum_{i} b_{i}^{2} f_{i}\right]^{-1}\left\{\sum_{i}\left(z_{s}^{i}\right)^{2} f_{i}+\frac{u^{\prime \prime}}{9}\left[\sum_{i}\left(z_{q}^{i}\right)^{2} f_{i} \sum_{j}\left(z_{s}^{j}\right)^{2} f_{j}-\left(\sum_{i} z_{q}^{i} z_{s}^{i} f_{i}\right]^{2}\right]\right\} \text {, } \\
& \frac{\partial \rho_{q}}{\partial \mu_{s}}=\left(1+u^{\prime \prime} \sum_{i} b_{i}^{2} f_{i}\right]^{-1}\left\{\sum_{i} z_{q}^{i} z_{s}^{i} f_{i}-\frac{u^{\prime \prime}}{9}\left[\sum_{i}\left(z_{q}^{i}\right)^{2} f_{i} \sum_{j}\left(z_{s}^{j}\right)^{2} f_{j}-\left[\sum_{i} z_{q}^{i} z_{s}^{i} f_{i}\right]^{2}\right]\right\}, \\
& \frac{\partial s}{\partial T}=\sum_{i}\left[h_{i}-d_{i}\left[z_{q}^{i} \frac{\mu_{q}-\Sigma / 3}{T}+z_{s}^{i} \frac{\mu_{s}-\Sigma / 3}{T}\right]\right] \\
& -\left(1+u^{\prime \prime} \sum_{i} b_{i}^{2} f_{i}\right]^{-1}\left\{\sum_{i} e_{i}\left[z_{q}^{i} \frac{\mu_{q}-\Sigma / 3}{T}+z_{s}^{i} \frac{\mu_{s}-\Sigma / 3}{T}\right)\right. \\
& +u^{\prime \prime}\left[\left(\sum_{i} b_{i} d_{i}\right]^{2}-\sum_{i} b_{i} d_{i} \sum_{j} b_{j} f_{j} \mid z_{q}^{j} \frac{\mu_{q}-\Sigma / 3}{T}+z_{s}^{j} \frac{\mu_{s}-\Sigma / 3}{T}\right] \\
& \left.\left.+\frac{1}{3} \sum_{i} z_{q}^{i} e_{i} \sum_{j} z_{s}^{j} b_{j} f_{j}\left[\frac{\mu_{q}-\mu_{s}}{T}\right)-\frac{1}{3} \sum_{i} z_{s}^{i} e_{i} \sum_{j} z_{q}^{j} b_{j} f_{j}\left(\frac{\mu_{q}-\mu_{s}}{T}\right)\right]\right\} \text {, } \\
& f_{i}=\frac{g_{i}}{2 \pi^{2} T} \int_{0}^{\infty} d k k^{2}\left(\bar{n}_{k}^{i}\right)^{2} \exp (\beta A), \quad d_{i}=\frac{g_{i}}{2 \pi^{2} T^{2}} \int_{0}^{\infty} d k k^{2} \sqrt{k^{2}+m_{i}^{2}}\left(\bar{n}_{k}^{i}\right)^{2} \exp (\beta A),
\end{aligned}
$$




$$
\begin{aligned}
& e_{i}=d_{i}-f_{i}\left[z_{q}^{i} \frac{\mu_{q}-\Sigma / 3}{T}+z_{s}^{i} \frac{\mu_{s}-\Sigma / 3}{T}\right], \quad h_{i}=\frac{g_{i}}{2 \pi^{2} T^{3}} \int_{0}^{\infty} d k k^{2}\left(k^{2}+m_{i}^{2}\right)\left(\bar{n}_{k}^{i}\right)^{2} \exp (\beta A), \\
& \bar{n}_{k}^{i}=\left[\exp (\beta A)+\theta_{i}\right]^{-1}, \quad A=\sqrt{k^{2}+m_{i}^{2}}-\left(z_{q}^{i} \frac{\mu_{q}-\Sigma / 3}{T}+z_{s}^{i} \frac{\mu_{s}-\Sigma / 3}{T}\right) .
\end{aligned}
$$

${ }^{1}$ For an overview, see J. Cleymans, R. V. Gavai, and E. Suhonen, Phys. Rep. 130, 217 (1986), and references therein.

${ }^{2}$ J. Engels, F. Karsch, I. Montvay, and H. Satz, Phys. Lett. 101B, 89 (1981); 102B, 332 (1981); J. Engels, F. Karsch, and H. Satz, Nucl. Phys. B205 [FS5], 239 (1982); F. Karsch, ibid. B205 [FS5], 285 (1982).

${ }^{3}$ W. Scheid, H. Müller, and W. Greiner, Phys. Rev. Lett. 32, 741 (1974).

${ }^{4}$ H. G. Baumgardt, J. U. Schott, Y. Sakamoto, E. Schopper, H. Stöcker, J. Hofmann, W. Scheid, and W. Greiner, Z. Phys. A 273, 359 (1975).

5 J. Hofmann, W. Scheid, and W. Greiner, Nuovo Cimento 33A, 343 (1976).

${ }^{6}$ L. van Hove, Z. Phys. C 21, 93 (1983); 27, 135 (1985).

${ }^{7}$ H. H. Gutbrod, K. H. Kampert, B. W. Kolb, A. M. Poskanzer, H. G. Ritter, and H. R. Schmidt, Phys. Lett. B 216, 267 (1989).

${ }^{8}$ H. Stöcker, Nucl. Phys. A418, 587c (1984).

${ }^{9}$ D. H. Rischke, B. L. Friman, H. Stöcker, and W. Greiner, J. Phys. G 14, 191 (1988).

${ }^{10}$ K. A. Bugaev and M. I. Gorenstein, Z. Phys. C 43, 261 (1989).

${ }^{11}$ H. W. Barz, L. P. Csernai, B. Kämpfer, and B. Lukács, Phys. Rev. D 32, 115 (1985).

${ }^{12}$ K. A. Bugaev, M. I. Gorenstein, B. Kämpfer, and V. I. Zhdanov, Phys. Rev. D 40, 2903 (1989).

${ }^{13}$ M. Gyulassy, K. Kajantie, H. Kurki-Suonio, and L. McLerran, Nucl. Phys. B237, 477 (1984).

${ }^{14}$ B. Kämpfer, H. W. Barz, L. Münchow, and B. Lukács, Acta Phys. (N.Y.) Pol. B 17, 685 (1986).

${ }^{15}$ B. L. Friman, G. Baym, and J. P. Blaizot, Phys. Lett. 132B, 291 (1983).

16J. D. Walecka, Ann. Phys. (N.Y.) 83, 491 (1974); J. Boguta and A. R. Bodmer, Nucl. Phys. A292, 413 (1977); B. M. Waldhauser, J. A. Maruhn, H. Stöcker, and W. Greiner, Phys. Rev. C 38, 1003 (1988).

${ }^{17}$ B. A. Freedman and L. D. McLerran, Phys. Rev. D 16, 1130 (1977); 16, 1147 (1977); 16, 1169 (1977).

${ }^{18}$ A. Chodos, R. L. Jaffe, K. Johnson, C. B. Thorn, and V. F. Weisskopf, Phys. Rev. D 9, 3471 (1974).

${ }^{19}$ J. I. Kapusta, Nucl. Phys. B148, 461 (1979).

${ }^{20}$ J. Cleymans, K. Redlich, H. Satz, and E. Suhonen, Z. Phys. C 33, 151 (1986).

${ }^{21}$ U. Heinz, P. R. Subramanian, H. Stöcker, and W. Greiner, J. Phys. G 12, 1237 (1986); C. Greiner, P. Koch, and H. Stöcker, Phys. Rev. Lett. 58, 1825 (1987).

${ }^{22}$ P. R. Subramanian, H. Stöcker, and W. Greiner, Phys. Lett. B 173, 468 (1986).

${ }^{23}$ R. Hagedorn and J. Rafelski, in Statistical Mechanics of Quarks and Hadrons, edited by H. Satz (North-Holland, Amsterdam, 1981), p. 237.

${ }^{24}$ H. W. Barz, B. L. Friman, J. Knoll, and H. Schulz, Phys. Rev. D 40, 157 (1989).

${ }^{25}$ J. M. Eisenberg and W. Greiner, Nuclear Theory I: Nuclear Models, 3rd ed. (North-Holland, Amsterdam, 1987).
${ }^{26}$ For the validity of this approximation see Ref. 9.

27J. Zimányi, B. Lukács, P. Lévai, J. P. Bondorf, and N. L. Balazs, Nucl. Phys. A484, 647 (1988).

${ }^{28}$ W. Scheid and W. Greiner, Z. Phys. 226, 364 (1969).

${ }^{29}$ In fact, this holds only for $C<9\left(m_{N}+B_{0}\right)=8307 \mathrm{MeV}$. In our calculations, however, $C \leq 5000 \mathrm{MeV}$, see below.

${ }^{30}$ D. Hahn and H. Stöcker, Nucl. Phys. A452, 723 (1986).

${ }^{31}$ J.-P. Blaizot, D. Gogny, and B. Grammaticos, Nucl. Phys. A292, 315 (1976).

${ }^{32}$ M. M. Sharma, in Nuclear Astrophysics, proceedings of the School, La Rabida, Spain, 1988, edited by M. Lozano, M. I. Gallardo, and J. M. Arias (Research Reports in Physics) (Springer, Berlin, 1989).

${ }^{33}$ In Ref. 34 it is explicitly shown that, with respect to the hadron-matter-QGP phase transition, the equation of state (10) yields quantitatively comparable results as the nonlinear $\sigma-\omega$ model for the hadronic part of the nuclear-matter equation of state.

${ }^{34}$ B. M. Waldhauser, D. H. Rischke, J. A. Maruhn, H. Stöcker, and W. Greiner, Z. Phys. C 43, 411 (1989).

${ }^{35}$ For the details of this fit see Ref. 9.

${ }^{36}$ L. D. Landau and E. M. Lifshitz, Fluid Mechanics (Pergamon, New York, 1959).

${ }^{37}$ B. L. Friman, K. Kajantie, and P. V. Ruuskanen, Nucl. Phys. B266, 468 (1986).

${ }^{38}$ A. M. Taub, Phys. Rev. 74, 328 (1948).

${ }^{39}$ P. Danielevicz and V. Ruuskanen, Phys. Rev. D 35, 344 (1987).

${ }^{40} \mathrm{R}$. Hagedorn, in Relativistic Kinematics (Benjamin, New York, 1963)

${ }^{41}$ M. I. Gorenstein and V. I. Zhdanov, Z. Phys. C 34, 79 (1987).

${ }^{42}$ Note, however, that the results of a hydrodynamic calculation of the expansion stage in a nucleus-nucleus collision, assuming a uniform mixed phase (Ref. 43), are well reproduced by a simulation, explicitly considering inhomogeneities in the mixed phase (Ref. 44).

${ }^{43}$ M. Kataja, P. V. Ruuskanen, L. McLerran, and H. von Gersdorff, Phys. Rev. D 34, 2755 (1986).

${ }^{44}$ G. Bertsch, M. Gong, L. McLerran, P. V. Ruuskanen, and E. Sarkkinen, Phys. Rev. D 37, 1202 (1988).

${ }^{45}$ Y. B. Zel'dovich and Y. P. Raizer, Physics of Shock Waves and High Temperature Hydrodynamic Phenomena (Academic, New York, 1966), Vols. I and II.

${ }^{46}$ Note that combustion is a spontaneous nonequilibrium process, thus rendering the system's evolution discontinuous in the space of the equilibrium variables $T$ and $\mu_{q}$; cf. Fig. 3 .

${ }^{47}$ H. W. Barz, B. L. Friman, J. Knoll, and H. Schulz, Nucl. Phys. A484, 661 (1988).

${ }^{48}$ In this work we do not consider possible solutions of the relativistic RHTA equation which correspond to shock surfaces with a timelike normal vector (Ref. 49).

${ }^{49}$ L. P. Csernai Zh. Eksp. Teor. Fiz. 92, 379 (1987) [Sov. Phys. JETP 65, 216 (1987)]; M. Gyulassy and L. P. Csernai, Nucl. Phys. A460, 723 (1986); L. P. Csernai and M. Gong, Phys. 
Rev. D 37, 3231 (1988).

${ }^{50}$ C. Greiner, D. H. Rischke, H. Stöcker, and P. Koch, Phys. Rev. D 38, 2797 (1988).

${ }^{51}$ B. M. Waldhauser, D. H. Rischke, B. L. Friman, J. A. Maruhn, H. Stöcker, and W. Greiner, GSI Report No. 88-1, 1987 (unpublished).

${ }^{52}$ WA 80 Collaboration, H. R. Schmidt, invited talk given at the Spring Meeting of Nuclear Physics Sections, Berlin, Germany, 1988 (unpublished).

${ }^{53}$ T. S. Biro, B. Lukács, and J. Zimanyi, Nucl. Phys. A386, 617
(1982).

${ }^{54}$ There are arguments (Refs. 8 and 55) that the nonequilibrium phase transition is to be regarded as a condensation discontinuity (Ref. 36).

${ }^{55} \mathrm{G}$. Baym (private communication).

${ }^{56}$ C. Greiner, D. H. Rischke, and H. Stöcker (in preparation).

${ }^{57}$ U. Heinz, Regensburg University Report No. TPR-88-33 (unpublished).

${ }^{58}$ P. Koch, B. Müller, H. Stöcker, and W. Greiner, Mod. Phys. A 3, 737 (1988). 